\title{
Proteomic analysis links alterations of bioenergetics, mitochondria-ER interactions and proteostasis in hippocampal astrocytes from 3xTg-AD mice
}

Giulia Dematteis ${ }^{1}$, Gabriele Vydmantaité2, Federico Alessandro Ruffinatti', Malak Chahin', Serena Farruggio ${ }^{3}$, Elettra Barberis ${ }^{3,4}$, Eleonora Ferrari ${ }^{4,5}$, Emilio Marengo ${ }^{6}$, Carla Distasi ${ }^{1}$, Ramune Morkūnienè ${ }^{7}$, Armando A. Genazzani ${ }^{1}$,

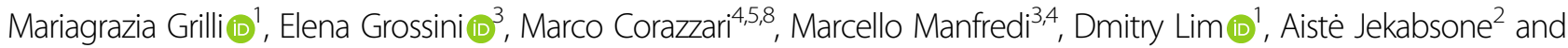
Laura Tapella (10 ${ }^{1}$

\begin{abstract}
The pathogenesis of Alzheimer's disease (AD), a slowly-developing age-related neurodegenerative disorder, is a result of the action of multiple factors including deregulation of $\mathrm{Ca}^{2+}$ homeostasis, mitochondrial dysfunction, and dysproteostasis. Interaction of these factors in astrocytes, principal homeostatic cells in the central nervous system, is still poorly understood. Here we report that in immortalized hippocampal astrocytes from 3xTg-AD mice (3Tg-iAstro cells) bioenergetics is impaired, including reduced glycolysis and mitochondrial oxygen consumption, and increased production of reactive oxygen species. Shotgun proteomics analysis of mitochondria-ER-enriched fraction showed no alterations in the expression of mitochondrial and OxPhos proteins, while those related to the ER functions and protein synthesis were deregulated. Using ER- and mitochondria-targeted aequorin-based $\mathrm{Ca}^{2+}$ probe we show that, in 3Tg-iAstro cells, ER was overloaded with $\mathrm{Ca}^{2+}$ while $\mathrm{Ca}^{2+}$ uptake by mitochondria upon ATP stimulation was reduced. This was accompanied by the increase in short distance $(\approx 8-10 \mathrm{~nm})$ contact area between mitochondria and ER, upregulation of ER-stress/unfolded protein response genes Atf4, Atf6 and Herp, and reduction of global protein synthesis rate. We suggest that familial AD mutations in 3Tg-iAstro cells induce mitochondria-ER interaction changes that deregulate astrocytic bioenergetics, $\mathrm{Ca}^{2+}$ homeostasis and proteostasis. These factors may interact, creating a pathogenic loop compromising homeostatic and defensive functions of astroglial cells predisposing neurons to dysfunction.
\end{abstract}

\footnotetext{
Correspondence: Dmitry Lim (dmitry.lim@uniupo.it) or Aistè. Jekabsone (Aiste. Jekabsone@lsmuni.lt)

'Department of Pharmaceutical Sciences, Università degli Studi del Piemonte Orientale, Novara, Italy

${ }^{2}$ Institute of Pharmaceutical Technologies, Faculty of Pharmacy, Medical Academy, Lithuanian University of Health Sciences, Kaunas, Lithuania

Full list of author information is available at the end of the article

These Authors contributed equally: Giulia Dematteis, Gabrielè Vydmantaitè

Edited by A. Verkhratsky
}

\begin{abstract}
Introduction
Alzheimer's disease (AD) is a devastating age-related neurodegenerative disorder with a complex and slowlydeveloping pathogenesis ${ }^{1}$. Modern hypotheses, aiming at explanation the mechanisms of $\mathrm{AD}$ development, link ageing with destructive effects of Familial Alzheimer Disease (FAD) mutations and $\beta$-amyloid (A $\beta$ ) burden. Among such hypotheses are the calcium hypothesis of neurodegeneration ${ }^{2}$ and the mitochondrial cascade hypothesis, the latter including also the oxidative stress
\end{abstract}


and the energy dysbalance hypotheses ${ }^{3,4}$. The $\mathrm{Ca}^{2+}$ hypothesis concerns the enhancement of neuronal $\mathrm{Ca}^{2+}$ signaling leading to excitotoxicity and neuronal death ${ }^{5}$, and the over-activation of calcineurin $(\mathrm{CaN})$ signaling $^{6}$ linked to altered neuronal plasticity and memory deficit. Mitochondrial dysfunction occurs early in $\mathrm{AD}^{7}$. The aging processes and FAD-associated mutations can promote mitochondrial dysfunctions in the central nervous system $(\mathrm{CNS})^{8-10}$. Alterations of oxidative phosphorylation determine the decrease of adenosine triphosphate (ATP) and the increase of reactive oxygen species (ROS) production accompanied by fatty acid peroxidation. Both oxidative stress and decrease in ATP strongly impact neuronal function by impairing all energy-demanding processes, affecting protein folding, and causing DNA damage ${ }^{11}$. Recent evidence indicates that $\mathrm{Ca}^{2+}$ and mitochondria can be tandem players in $\mathrm{AD}$ development. A significant reduction in expression of mitochondrial $\mathrm{Ca}^{2+}$ efflux controlling proteins is observed in prefrontal cortex of both $3 x \mathrm{Tg}-\mathrm{AD}$ mice models and human individuals with $\mathrm{AD}^{12}$. Moreover, the study provides substantial experimental evidence that mitochondrial $\mathrm{Ca}^{2}$ + overload contributes to AD progression by promoting mitochondrial dysfunction, superoxide generation, and neuronal cell death. Mitochondria-endoplasmic reticulum (mito-ER) interaction has recently been suggested to play an important role in cellular physio-pathology ${ }^{13,14}$. Studies suggest that mito-ER interaction is altered in fibroblasts from FAD patients ${ }^{15}$ and in cellular AD models ${ }^{16}$. Forcing mito-ER tethering in a Drosophila model of AD increased lifespan of model animals ${ }^{17}$. These findings were focused and hypotheses considered principally for the neuronal dysfunction, while their application for astroglial cells has not been studied in details.

Astrocytes are fundamental homeostatic cells in the CNS. They provide structural, metabolic, and signaling support to neurons ${ }^{18,19}$. Growing body of evidence suggests that, during $\mathrm{AD}$ pathogenesis, astrocytic dysfunction may precede or be parallel to the neuronal dysfunction ${ }^{20-22}$. In this aspect, mitochondrial function in astrocytes is of
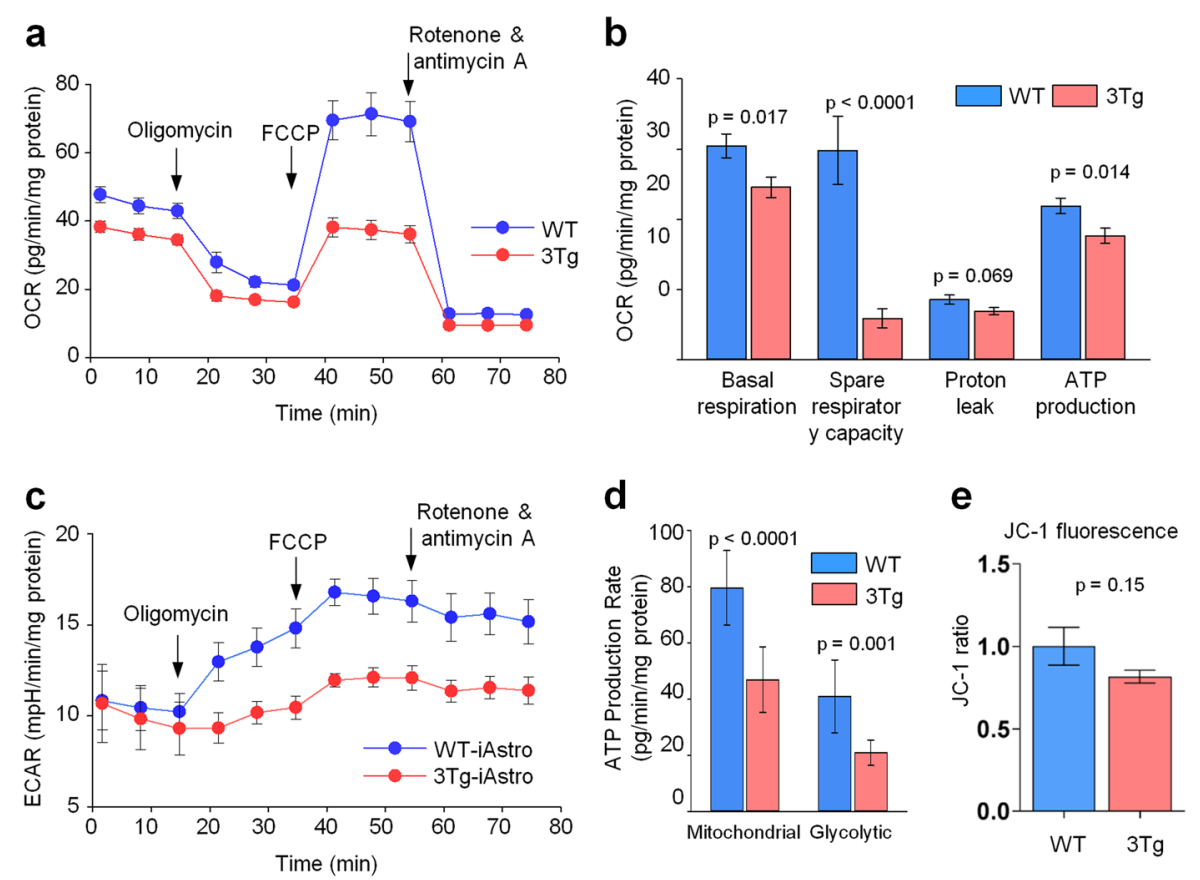

Fig. 1 Mitochondrial and glycolytic energy metabolism is impaired in 3Tg-iAstro compared with WT-iAstro cells. Bioenergetics of iAstro cells were assessed by Seahorse Flux Analyzer using Cell Mito Stress Kit. In $\mathbf{a}$, there are mitochondrial oxygen consumption curves presented as averages \pm standard deviations of each measurement time point $(n=9)$. Initial three measurement points represent basal mitochondrial respiration, the next three points after blocking adenine nucleotide translocating by adding oligomycin represent proton leak-stimulated oxygen consumption, then follow three points representing maximal mitochondrial oxygen consumption capacity when mitochondrial inner membrane is uncoupled by FCCP, and the last three points are for non-mitochondrial oxygen consumption when mitochondrial respiratory chain is inhibited by rotenone and antimycin A (see more detailed explanation on manufacturer's website https://www.agilent.com/en/products/cell-analysis/seahorse-xf-consumables/ kits-reagents-media/seahorse-xf-cell-mito-stress-test-kit). The summary data calculated from the curves in a are shown in $\mathbf{b}$. In $\mathbf{c}$ representing efficiency of glycolysis, there are curves of $\mathrm{pH}$ changes in the cell culture medium measured simultaneously with oxygen consumption rate. Section (d) shows mitochondrial and glycolytic ATP production in iAstro cells assessed by Seahorse Flux Analyzer using Real-Time ATP Rate Assay ( $n=9$ ). e Mitochondrial membrane potential was measured using JC-1 fluorescent ratiometric probe on $n=3$ independent cultures assayed in quadriplicates $(p=0.15)$. 
special interest as it may specifically be involved in deregulation of synaptic transmission ${ }^{23}$. While the role of astrocytic mitochondria in AD is being now acknowledged $^{24,25}$, it appears difficult to dissect the astrocytic versus neuronal mitochondrial dysfunction in $\mathrm{AD}$ brain, and the knowledge about astrocyte-specific mitochondrial alterations, their link to astrocytic $\mathrm{Ca}^{2+}$ signaling and other cellular processes as endoplasmic reticulum (ER)stress proteostasis remains limited ${ }^{26}$.

Recently, we have generated and characterized immortalized astrocytic cell lines from hippocampi of 3xTg-AD mice, a popular and well-studied AD mouse model ${ }^{27}$. These lines, named WT- and 3xTg-AD immortalized astrocytes (WT-iAstro and 3Tg-iAstro) recapitulate the features of primary astrocytic cultures from $3 \times \mathrm{Tg}-\mathrm{AD}$ mice in terms of gene profiling, protein expression and $\mathrm{Ca}^{2+}$ signaling ${ }^{27,28}$. Here we used WT-iAstro and 3TgiAstro lines to study mitochondrial functions and their association with $\mathrm{Ca}^{2+}$ signaling, mito-ER interaction and proteostasis. Our data suggest that the functional impairment of mitochondrial respiration in FAD astrocytes may be associated with deregulations of cellular $\mathrm{Ca}^{2+}$ homeostasis and protein synthesis through altered mitochondria-ER interaction.

\section{Results}

\section{Tg-iAstro astrocytes have impaired ATP synthesis and mitochondrial functions}

Investigation of metabolic activity by Seahorse XF Cell Mito Stress Assay revealed significant decrease in basal mitochondrial oxygen consumption rate (OCR) of $3 \mathrm{Tg}$ iAstro cells compared to the WT-iAstro line (Fig. 1a, b). The average OCR in 3Tg-iAstros was 19\% lower than in astrocytes without $\mathrm{AD}$ mutations. The same tendency remained for the mitochondrial OCR sensitive to ATPase inhibitor oligomycin; ATP production-coupled OCR in 3Tg-iAstro cells was also by $19 \%$ lower than in WT-iAstro cells. Moreover, when mitochondria were stressed by permeabilizing inner membrane for $\mathrm{H}^{+}$with carbonyl cyanide 4-(trifluoromethoxy)phenylhydrazone (FCCP) to reveal maximal mitochondrial respiratory capacity, the responsive increase in OCR of 3Tg-iAstro cell mitochondria was five times lower compared to that in healthy WT-iAstro cells (see OCR after addition of FCCP in Fig. 1a and Spare respiratory capacity bars in Fig. 1b). There was no significant difference in proton leak-driven OCR observed between 3Tg and WT-iAstro cell lines.

Glycolysis activity of the cells was assessed as extracellular acidification rate (ECAR) simultaneously with the OCR in the same samples. There was no difference in ECAR between the two cell lines when the cells were not stressed by mitochondrial inhibitors (Fig. 1c). After blocking phosphorylation by oligomycin, the ECAR induced by WT-iAstro cells started to increase indicating glycolysis activation to restore energetic balance. However, in the probes containing 3Tg-iAstro cells, this increase was significantly lower. The impairment of both mitochondrial and glycolytic energy metabolism in 3TgiAstro cells was also confirmed by Seahorse XF Real-Time ATP Rate Assay (Fig. 1d). Compared to the WT-iAstro cells, the average decrease in calculated ATP amount produced by mitochondrial and glycolytic pathways was $41 \%$ and $49 \%$, respectively.

Altered OCR and ECAR may be associated with impairment of mitochondrial membrane potential $(\Delta \psi \mathrm{m})$. However, we found that, although there was a trend to a lower $\Delta \psi \mathrm{m}$ in 3Tg-iAstro cells, the difference was not significant $(81.67 \pm 3.87 \%$ of WT-iAstro, $p=0.15)$ (Fig. 1e).

Increased mitochondrial ROS production in 3Tg-iAstro cells

Mitochondrial dysfunction is usually related with ROS production, and both features are characteristic to neuronal pathology in AD. To investigate if mitochondrial failure is also accompanied by ROS generation in hippocampal astrocytes from AD mouse model, we have compared ROS levels in 3Tg-iAstro and WT-iAstro cells. Assessment of intracellular hydroxyl, peroxyl and other ROS by DCFDA assay revealed significant increase of the species inside 3Tg-iAstro compared to WT-iAstro cells (Fig. 2a, c, d). The difference in the level of 2,7-dichlorofluorescein (DCF) fluorescence between 3Tg-iAstro and WT-iAstro samples increased during the first $30 \mathrm{~min}$ of monitoring in a plate reader, and was by $76 \%$ higher in 3Tg-iAstro cells at this time point (Fig. 2c). The fluorescence intensity in 3Tg-iAstro cells remained higher by $78 \%$ and $85 \%$ after 60 and 90 min, respectively. Similarly, $73 \%$ higher DCF fluorescence intensity in 3Tg-iAstro cells compared to WT-iAstro samples was found by evaluation of cell fluorescence intensity in microscope images by ImageJ software (Fig. 2d).

The primary ROS produced within mitochondria is superoxide, most of which is converted to hydrogen peroxide by superoxide dismutase ${ }^{29}$. Hydrogen peroxide is membrane permeable and freely diffuses to cytosol where it might be further converted to other ROS such as hydroxyl radical by Fenton reaction. On the contrary, superoxide cannot pass membranes and remains at the production site. If the source of cytosolic ROS is mitochondrial hydrogen peroxide, the levels of mitochondrial superoxide should also be increased. Indeed, images of 3Tg-iASTRO cells loaded with mitochondrially targeted fluorescent superoxide indicator MitoSOX ${ }^{\mathrm{TM}}$ Red had more intensively stained mitochondrial network compared to WT-iAstro cells (Fig. 2b, Supplementary Fig. 1). Quantitative evaluation of the images revealed 63\% higher MitoSOX fluorescence in 3Tg-iAstro cells (Fig. 2e). This was equal to the level induced by mitochondrial 
a

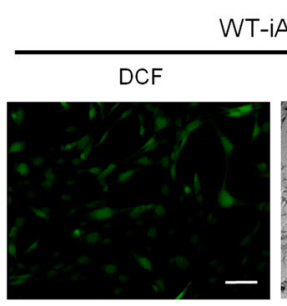

b
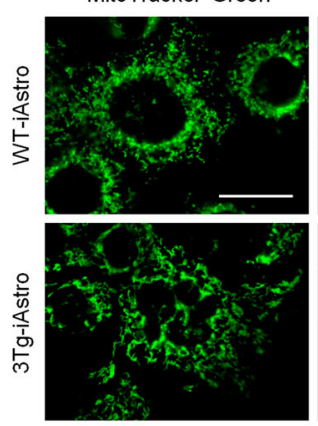

C

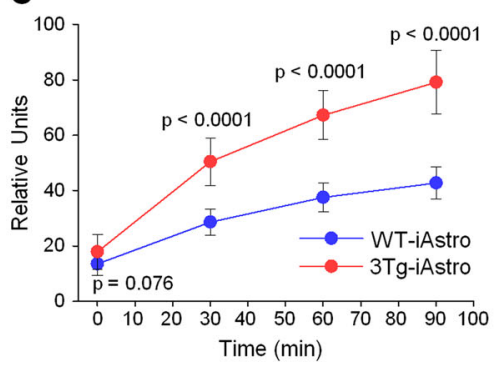

MitoSOX
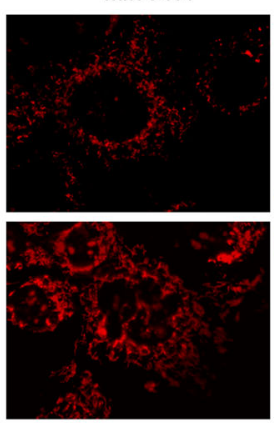

d

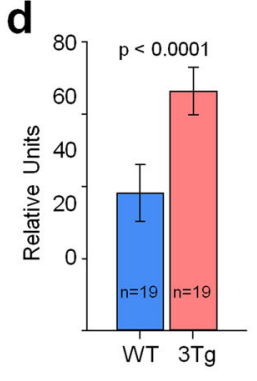

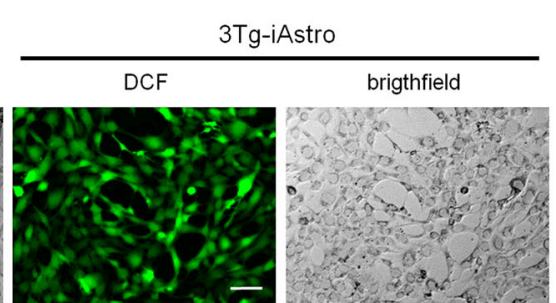

Hoechst
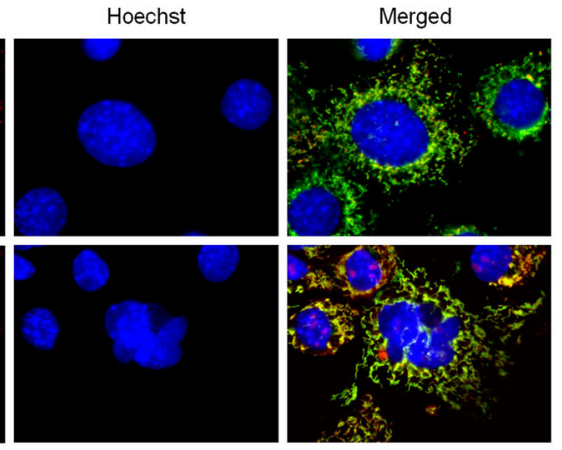

e

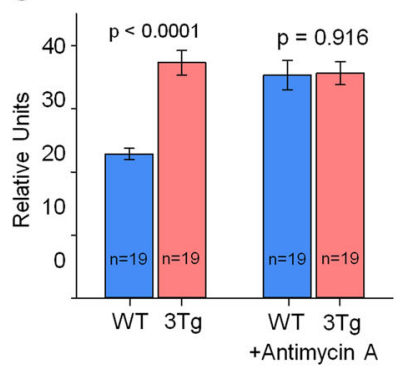

Fig. 2 Intracellular ROS and intra-mitochondrial superoxide production are increased in $\mathbf{3 T g}$-iAstro vs WT-iAstro cells. Panels a, $\mathbf{c}$, and $\mathbf{d}$ show 2',7'-dichlorofluorescein (DCF) fluorescence in 2',7'-dichlorofluorescin diacetate (DCFDA)-treated iAstro cells. In a, the bright-field images of the same fields are presented next to the fluorescent images to show the total cell number in the field, scale bar is $100 \mu \mathrm{m}$. Panel c demonstrates DCF fluorescence in iAstro cells measured at several time points after leading with DCFDA (mean \pm SD of seven biological replicates) in a fluorometric plate reader. In $\mathbf{d}$, the fluorescence intensity of the cell covered area in micrographs was assessed by ImageJ software (Huang algorithm). Panels $\mathbf{b}$ and $\mathbf{c}$ show MitoSOX fluorescence upon binding mitochondrial superoxide. In $\mathbf{b}$, there are representative images of total mitochondrial network images visualized by MitoTracker Green and mitochondrial superoxide radicals visualized by MitoSOX, scale bar is $50 \mu \mathrm{m}$. The quantitative data for MitosOX fluorescence are presented in panel e.

respiratory complex III inhibitor $100 \mu \mathrm{M}$ Antimycin A, a well-known inducer of mitochondrial superoxide, which was applied as a positive control of the assay.

Proteomic analysis of MERE fraction from WT- and 3TgiAstro lines shows absence of protein expression alterations in mitochondria

Next, we thought to investigate if the impairment of cellular bioenergetics was a result of altered expression of protein components including glycolysis and mitochondrial OXPHOS pathway as it was recurrently suggested for $\mathrm{AD}^{30-32}$. For this, we have isolated Mitochondria/ Endoplasmic Reticulum-Enriched (MERE) fractions and performed shotgun mass spectrometry (SG-MS) proteomics followed by bioinformatic analysis (Fig. 3a). 1089 and 928 proteins have been identified in WT-iAstro and 3Tg-iAstro lines, respectively, with 777 proteins common for WT and 3Tg samples (Supplementary Table 1a). Quantification of proteins resulted in 53 differentially expressed proteins (DEPs) in 3Tg-iAstro vs WT-iAstro cells (Fig. $3 \mathrm{~b}$ and Supplementary Table 1b). Comparison with our previously published proteome dataset obtained on whole-cell lysates from same WT-iAstro and 3TgiAstro cells ${ }^{27}$ showed 489 common proteins between MERE fraction and whole-cell lysate, while only six DEPs were found to be common between MERE fractions and whole-cell preparations (Fig. 4a). Next, we analyzed the sub-cell compartment composition of identified and DEPs 


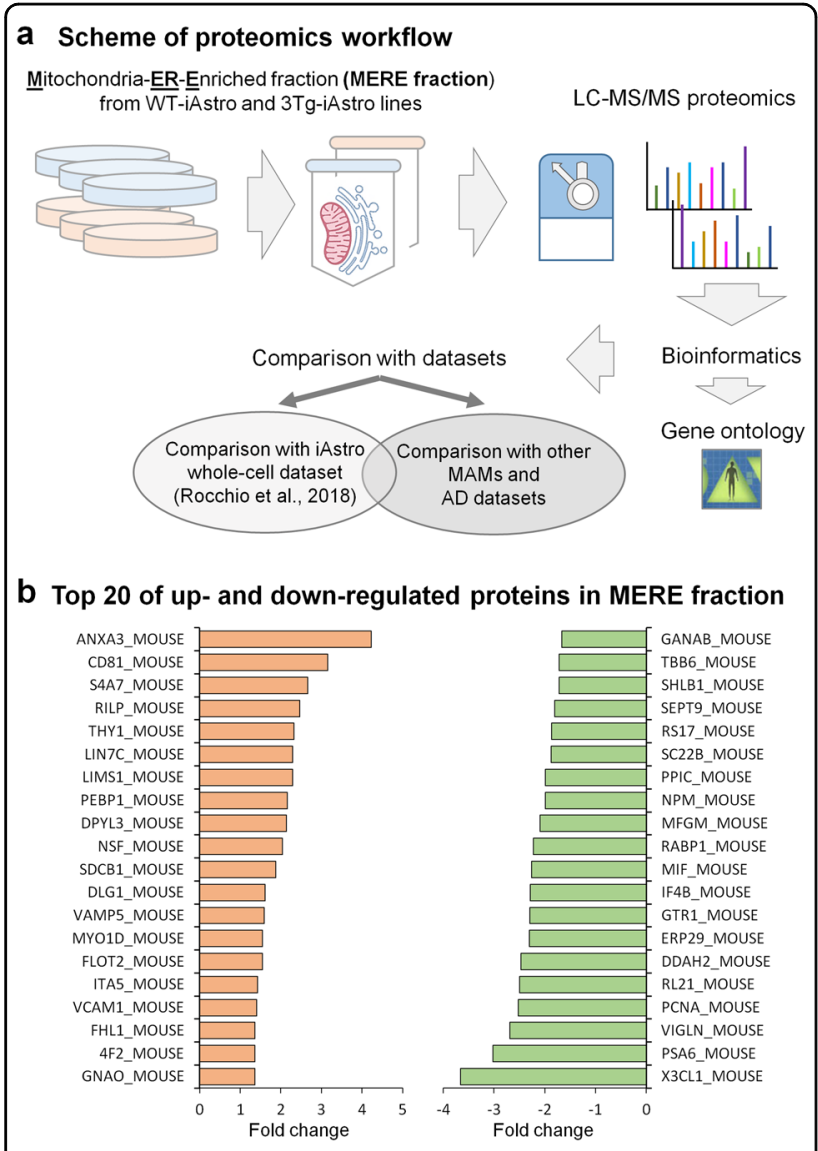

Fig. 3 Mass spectrometry proteomic analysis of mitochondria-ERenriched fractions form WT- and 3Tg-iAstro lines. a Cartoon shown workflow of proteomic and bioinformatic analysis of mitochondria-ER-enriched (MERE) fractions form WT- and 3Tg-iAstro lines. $\mathbf{b}$ Top 20 of up- and downregulated differentially expressed proteins (DEPs).

proteins in MERE fraction in comparison with whole-cell dataset ${ }^{27}$. For this, proteins were assigned to one of the following ten groups according to Genecards database (https://www.genecards.org/): cytosol, plasma membrane, mitochondria, ER, Golgi apparatus (GA), intracellular vesicles, ribosomes, cytoskeleton, nucleus, and extracellular proteins. In the identified proteins list we found near twofold enrichment of mitochondrial and ER proteins and three-fold enrichment of GA proteins in MERE fraction compared with whole-cell proteins with $30 \%$ decrease of PM and the absence of extracellular proteins (Fig. 4b). Surprisingly, in MERE fraction DEPs, there was only one mitochondrial protein, dimethylarginine dimethylaminohydrolase 2 (Uniprot_ID DDAH2_MOUSE, mouse gene Ddah2). Instead, there were 43 and 33\% more ER and GA proteins, respectively (Fig. 4c). Cytosolic, intracellular vesicle, ribosomal, cytoskeletal, and nuclear proteins were less abundant (by $30 \%, 59 \%, 79 \%, 54 \%$, and $77 \%$, respectively). Of note, components of the glycolytic pathway were absent in DEPs of MERE fraction, while whole-cell lysate DEPs list contained two glycolytic enzymes, namely fructose-bisphosphate aldolase A (Aldoa, 1.47 fold change) and of pyruvate kinase isoform M1 (Pkm, -2.21 fold change).

\section{Bioinformatic analysis of MERE fraction and whole-cell lysate DEPs from WT- and 3Tg-iAstro lines shows alterations in adhesion, ER and protein synthesis}

To investigate the functional significance of protein expression alterations we merged MERE fraction DEPs with those of whole-cell lysates. DAVID gene ontology analysis of 120 DEPs of the merged list resulted in 81 significantly overrepresented GO terms. Top overrepresented GO terms were as follows: Biological Processes - translation, cell-cell adhesion, and protein folding; among Cell Component terms - myelin sheath, focal adhesion, intracellular ribonucleoprotein complex, cell-cell adherens junction, and ribosome; among Molecular Function terms - poly(A) RNA binding, cadherin binding involved in cell-cell adhesion, RNA binding, structural constituent of ribosome, protein binding, unfolded protein binding. The overrepresented KEGG pathway was Ribosome (Fig. 5 and Supplementary Table 2).

\section{Poor overlap with MAMs and mitochondrial datasets from mouse AD models}

Next, we compared MERE fraction and whole cells 3TgiAstro datasets with mitochondria-associated membranes (MAMs) and mitochondrial proteome datasets reported by Völgyi et al. ${ }^{33}$ and Yu et al. ${ }^{31}$, respectively. Völgyi et al. reported deregulation of many ribosomal proteins in MAMs from 3-month-old APP/PS1 AD mouse. However, there were only two proteins common with our MERE fraction dataset: oppositely regulated ribosomal protein 21 (Rpl21) and co-downregulated Syntenin-1 (Sdcbp) (Supplementary Table 3a, b). Dataset by Yu et al. features mitochondrial proteome from hippocampus and cortex of symptomatic 15-month-old 3xTg-AD mice, the same from which $3 \mathrm{Tg}$-iAstro originated. Also in this case two protein were common with our MERE fraction dataset: co-downregulated vacuolar proton pump subunit B2 (Atp6v1b2) and oppositely regulated vesicular-fusion ATPase NSF (Supplementary Table 3c, d).

Taken together, these results suggest that the expression of mitochondrial proteins, which have been identified in MERE fractions, was largely unaltered in WT-iAstro and 3Tg-iAstro lines. Instead, the protein expression alterations are mainly related to ER and protein synthesis and processing. We reasoned that, in terms of association with protein expression, OXPHOS deficiency in 3TgiAstro cells may be associated with extra-mitochondrial factors. Therefore, we decided to focus on investigation of the following aspects: (i) relationships between $\mathrm{Ca}^{2+}$ 
a

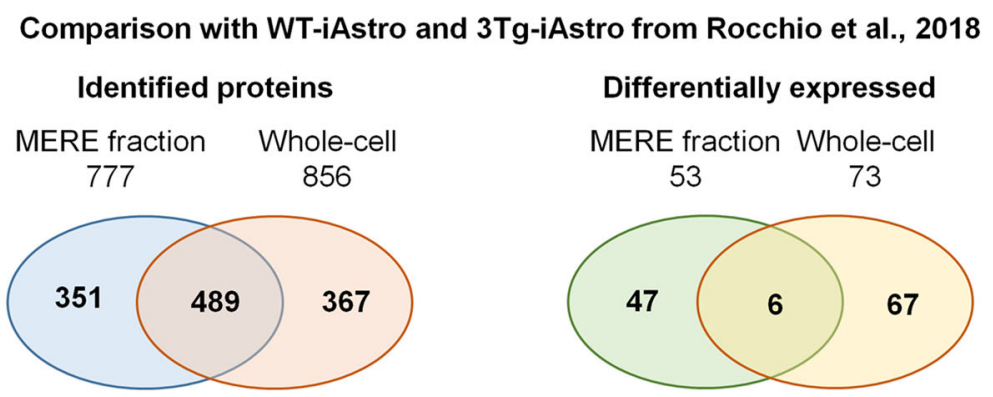

b Cell Component analysis of identified proteins
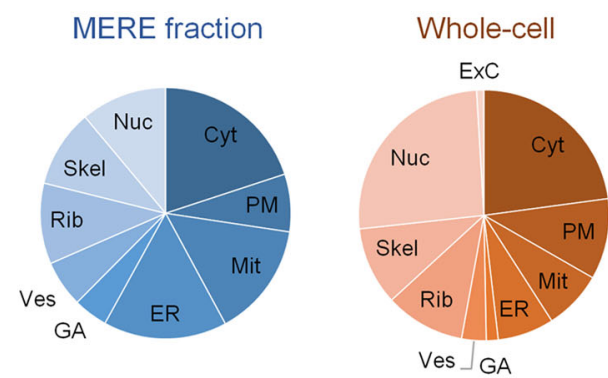

\begin{tabular}{|l|l|c|c|c|c|}
\hline & & \multicolumn{2}{|c|}{ MERE fraction } & \multicolumn{2}{c|}{ Whole-cell } \\
\hline Abbr & Cell component & Proteins & $\%$ & Proteins & $\%$ \\
\hline Cyt & Cytosol & 153 & 19.7 & 195 & 22.8 \\
\hline PM & Plasma membrane & 57 & 7.3 & 89 & 10.4 \\
\hline Mit & Mitochondria & 113 & 14.5 & 65 & 7.6 \\
\hline ER & Endoplasmic reticulum & 122 & 15.7 & 62 & 7.2 \\
\hline GA & Golgi apparatus & 34 & 4.4 & 13 & 1.5 \\
\hline Ves & Vesicels & 46 & 5.9 & 27 & 3.2 \\
\hline Rib & Ribosomes & 80 & 10.3 & 88 & 10.3 \\
\hline Skel & Cytoskeleton & 77 & 9.9 & 86 & 10.0 \\
\hline Nuc & Nucleus & 85 & 10.9 & 220 & 25.7 \\
\hline ExC & Extracellular & - & - & 8 & 0.9 \\
\hline
\end{tabular}

C

Cell Component analysis of DEPs
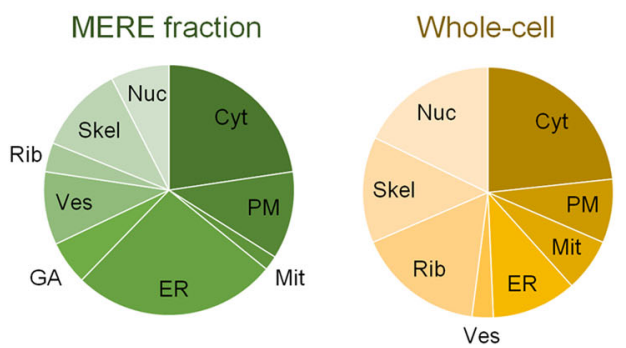

Fig. 4 Comparison of proteomic analyses of mitochondria-ER-enriched fractions and previously published whole-cells proteomics of WTiAstro vs 3Tg-iAstro cells. a comparison of identified and differentially expressed proteins in MERE fractions (this study) and whole-cell proteomics from WT- and 3Tg-iAstro cells. Cellular component analysis of identified (b) and differentially expressed proteins (c) in MERE fractions and whole-cell proteomics from WT-iAstro and 3Tg-iAstro cells.

signaling in the cytosol, ER and mitochondria; (ii) mitochondria-ER interaction; and (iii) assessment of the ER-stress/UPR and protein synthesis in 3Tg-iAstro vs WT-iAstro cells.

\section{Mitochondrial $\mathrm{Ca}^{2+}$ signaling is inhibited in $3 \mathrm{Tg}$-iAstro compared with WT-iAstro cells}

First, we investigated if ATP-induced mitochondrial $\mathrm{Ca}^{2+}$ signals in 3Tg-iAstro cells in comparison with WTiAstro cells were different from those detected in the cytosol and if the levels of $\mathrm{Ca}^{2+}$ in the ER may be associated with cytosolic and mitochondrial $\mathrm{Ca}^{2+}$ elevations. In the previous report we found that ATP-induced $\mathrm{Ca}^{2+}$ signals in 3Tg-iAstro lines were augmented as compared with WT-iAstro cells ${ }^{27}$. Here, using Fura- $2 \mathrm{Ca}^{2+}$ imaging we confirm this finding both in terms of the amplitude of
$\mathrm{Ca}^{2+}$ increase $(1.18 \pm 0.028$ norm. Fura-2 ratio for $3 \mathrm{Tg}$ iAstro, $n=63$, vs $1.48 \pm 0.047$ for 3Tg-iAstro, $n=69, p<$ $0.0001)$ and of the area under the $\mathrm{Ca}^{2+}$ trace $(33.69 \pm 1.73$ arbitrary units for 3Tg-iAstro, $n=63$, vs $60.61 \pm 3.11$ for 3Tg-iAstro, $n=69, p<0.0001$ ) (Fig. 6a). Next, using ERtargeted aequorin $\mathrm{Ca}^{2+}$ sensor $\mathrm{ER}-\mathrm{AEQ}^{34}$, we assessed whether the ATP-induced cytosolic $\mathrm{Ca}^{2+}$ transient may be associated with an augmented ER $\mathrm{Ca}^{2+}$ load. As shown in Fig. 6b, the steady-state ER luminal $\mathrm{Ca}^{2+}$ level was significantly higher in 3Tg-iAstro cells $(136.8 \pm 5.65 \mu \mathrm{M}$, $n=16)$ as compared with WT-iAstro cells (109.7 \pm $5.66 \mu \mathrm{M}, n=16, p=0.002$ ), confirming the higher ER $\mathrm{Ca}^{2+}$ load hypothesis. After this, employing MIT-AEQ aequorin $\mathrm{Ca}^{2+}$ sensor targeted to the mitochondrial matrix $^{34}$ we assessed ATP-evoked mitochondrial $\mathrm{Ca}^{2+}$ transients. Figure $6 \mathrm{c}$ shows that ATP-induced $\mathrm{Ca}^{2+}$ 


\begin{tabular}{|c|c|c|c|}
\hline \multicolumn{2}{|c|}{ GO category: Biological Process } & \multicolumn{2}{|c|}{ GO category: Molecular Function } \\
\hline GO Term & Adj.p.v & GO Term & Adj.p.v \\
\hline Translation & $9.8 \mathrm{E}-12$ & Poly(A) RNA binding & $1.85 \mathrm{E}-21$ \\
\hline Cell-cell adhesion & 0.0016 & \multirow{2}{*}{$\begin{array}{l}\text { Cadherin binding involved in } \\
\text { cell-cell adhesion }\end{array}$} & \multirow{2}{*}{$4.60 \mathrm{E}-13$} \\
\hline Protein folding & 0.0055 & & \\
\hline Substantia nigra development & 0.022 & \multirow{2}{*}{$\begin{array}{l}\text { Structural constituent of } \\
\text { ribosome }\end{array}$} & 2.11E-07 \\
\hline Regulation of cell shape & 0.047 & & 2.31E-07 \\
\hline \multicolumn{2}{|c|}{ GO category: Cellular Component } & Protein binding & 5.79E-07 \\
\hline GO Term & Adj.p.v & Unfolded protein binding & $3.76 \mathrm{E}-05$ \\
\hline Myelin sheath & $6.92 \mathrm{E}-23$ & \multicolumn{2}{|c|}{ GO category: Uniprot-Keywords } \\
\hline Focal adhesion & 4.91E-18 & GO Term & Adj.p.v \\
\hline $\begin{array}{l}\text { Intracellular ribonucleoprotein } \\
\text { complex }\end{array}$ & $1.79 \mathrm{E}-15$ & Ribonucleoprotein & 3.04E-11 \\
\hline Cell-cell adherens junction & $1.88 \mathrm{E}-14$ & Ribosomal protein & 1.96E-09 \\
\hline Melanosome & $1.83 \mathrm{E}-12$ & RNA-binding & $6.87 \mathrm{E}-08$ \\
\hline Ribosome & 4.19E-09 & Ubl conjugation & $7.88 \mathrm{E}-08$ \\
\hline \multicolumn{2}{|c|}{ GO category: KEGG Pathway } & Elongation factor & 1.18E-04 \\
\hline GO Term & Adj.p.v & Protein biosynthesis & $2.36 \mathrm{E}-04$ \\
\hline Ribosome & 1.69E-07 & & \\
\hline
\end{tabular}

Fig. 5 Analysis of protein-protein interaction network in merged DEPs list from MERE fractions and whole-cell proteomics from 3Tg-iAstro vs WT-iAstro cells using DAVID tool. List of 120 DEPs of joined lists of MERE fractions and whole-cell lysates from 3Tg-iAstro vs WT-iAstro cells was subjected to DAVID gene ontology tool analysis. The most significantly overrepresented specific GO terms are listed for Biological Process, Cellular Component, Molecular Function, Uniprot-Keywords, and KEGG Pathway categories. Complete GO analysis results are provided in Supplementary Table 2.

uptake in 3Tg-iAstro mitochondria was significantly lower than that in WT-iAstro mitochondria $(20.41 \pm$ $0.98 \mu \mathrm{M}, n=20$ vs $25.01 \pm 1.47 \mu \mathrm{M}, n=20$, respectively, $p=0.013)$. These results indicate that, in spite of the augmented ER $\mathrm{Ca}^{2+}$ content and increased ATP-induced cytosolic $\mathrm{Ca}^{2+}$ release, mitochondria in 3Tg-iAstro cells are defective in up-taking $\mathrm{Ca}^{2+}$ in comparison with WTiAstro cells.

\section{Shortened mitochondrial-ER distance in 3Tg-iAstro cells}

The deficient mitochondrial $\mathrm{Ca}^{2+}$ signaling, among other reasons, may be mediated by the alterations in the relationships between mitochondria and $\mathrm{ER}^{35}$. We have investigated this exploiting the recently generated SPLICS probe which allows visualizing mitochondrial-ER interaction point at two defined distances: $\approx 8-10 \mathrm{~nm}$ (SPLICSShort) and $\approx 40-50 \mathrm{~nm}$ (SPLICS-Long) ${ }^{15}$. In WT-iAstro cells SPLICS-Short was visualized as fluorescent dots localized mainly in the perinuclear zone (Fig. 7a). In 3TgiAstro cells SPLICS-Short expression resulted in a significantly higher number of fluorescent dots some of them appeared to be fused. Quantification of fluorescence area normalized for cell surface showed near doubling of the short-distance mito-ER contacts in 3Tg-iAstro cells as compared with WT-iAstro cells $(0.055 \pm 0.0037$ SPLICS/ cell ratio vs $0.031 \pm 0.0032$ SPLICS/cell ratio, respectively, $p<0.0001$ ) (Fig. 7a). SPLICS-Long expression in both WT-iAstro and 3Tg-iAstro cells was visualized as structures with diverse morphology, from dots of different size to tubular structures reminiscent shape of mitochondria. Overall, the SPLICS-Long fluorescence area in WT-iAstros was significantly higher than that of SPLICSShort $(p<0.0001)$. Nevertheless, the difference in SPLICSLong/cell area ratio between 3Tg-iAstro and WT-iAstro cells was not significative $(0.084 \pm 0.008$ SPLICS/cell ratio vs $0.069 \pm 0.0062$ SPLICS/cell ratio, respectively, $p=0.15$ ) (Fig. $7 \mathrm{~b}$ ) indicating that specifically short distance mitoER interaction is augmented in 3Tg-iAstro cells.

\section{Activation of unfolded-protein response and reduced protein synthesis in 3Tg-iAstro compare to WT-iAstro cells}

Proteomic analysis suggests that protein synthesis and processing may be impaired in 3Tg-iAstro cells. Therefore, we first measured mRNA levels of ER-stress and UPR-related gene. Real-time PCR analysis shows that Atf4, Atf6, and Herp mRNA were significantly upregulated in 3Tg-iAstro cells by $136 \%(p=0.037), 73 \%(p=$ $0.020)$ and $166 \%(p=0.031)$, respectively (Fig. 8a). $\mathrm{Xbp} 1$ showed a trend of upregulation (by $88 \%$ ) although the difference failed to reach significance $(p=0.059)$. To investigate protein translation efficiency, we used a method of surface sensing of translation (SUnSET) ${ }^{36,37}$. After puromycin pulsing of WT and Tg-iAstro we observed a $20 \% \quad(p=0.033)$ decrease of puromycin incorporation in 3Tg-iAstro compare to WT-iAstro (Fig. $8 b, c)$. 

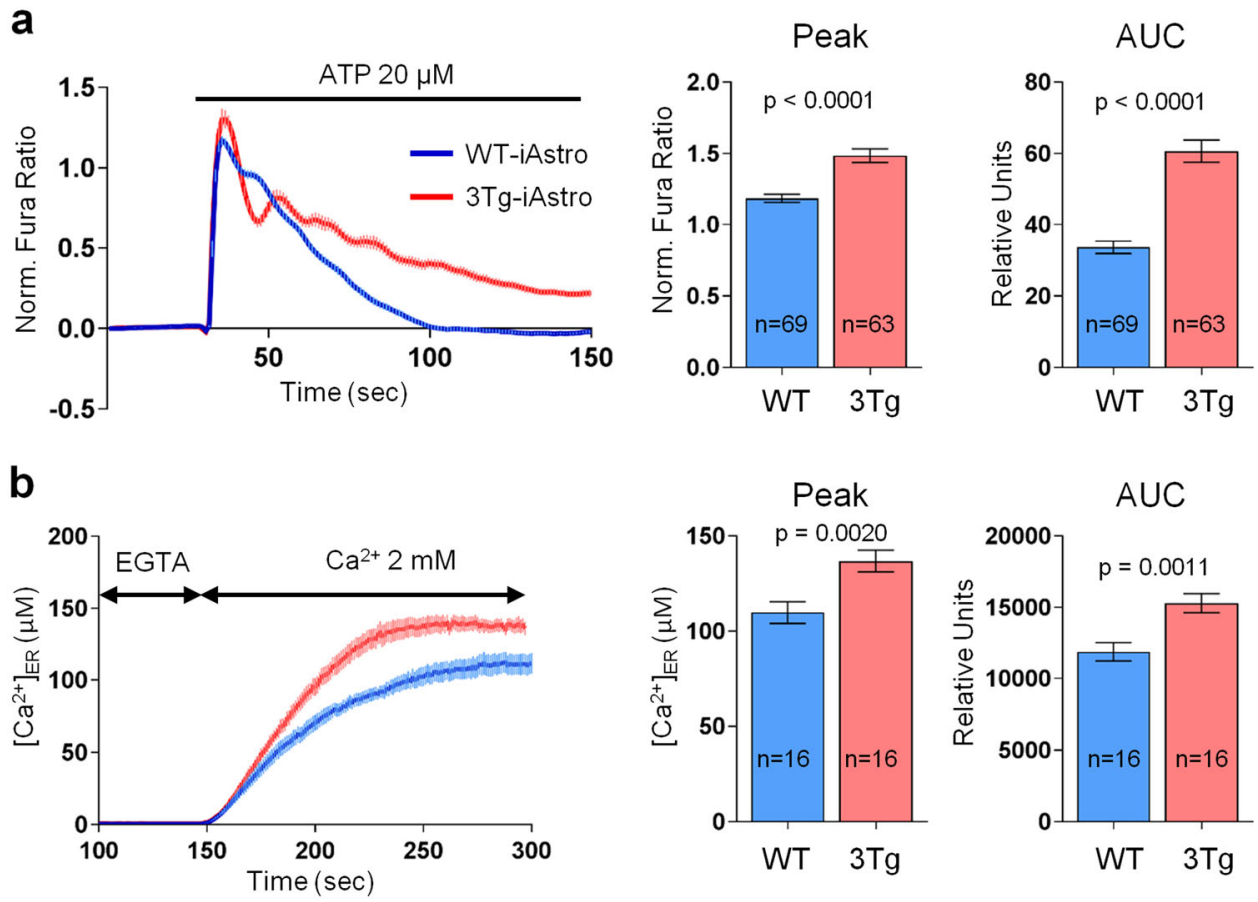

\section{C}
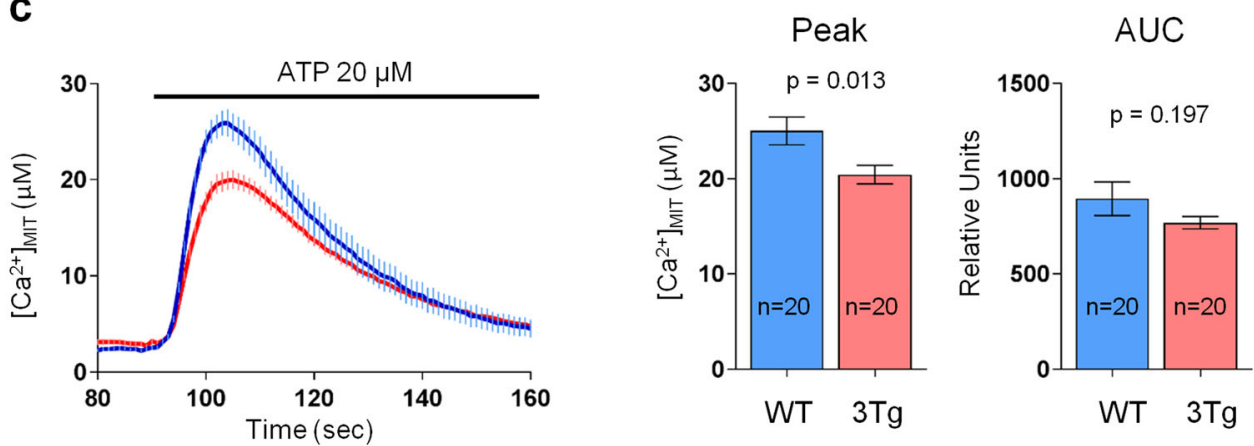

Fig. 6 Mitochondrial $\mathrm{Ca}^{2+}$ signaling is impaired in 3Tg-iAstro as compared with WT-iAstro cells. a WT- and 3Tg-iAstro cells, previously loaded with Fura-2, were stimulated with $20 \mu \mathrm{M}$ ATP in $\mathrm{Ca}^{2+}{ }^{2}$-containing KRB solution. Data are expressed as mean of peak \pm SEM $(p<0.0001)$ or mean and of area under the curve (AUC) \pm SEM $(p<0.0001)$ of $69 \mathrm{WT}$-iAstro cells and $633 \mathrm{Tg}$-iAstro cells from nine coverslips from three independent experiments. b WT- and 3Tg-iAstro cells, stably expressing ER-AEQ $\mathrm{Ca}^{2+}$ sensor, were reconstituted with coelenterazine $n$ (low light-emitting coelenterazine) in KRB solution supplied with $600 \mu \mathrm{M}$ EGTA and $3 \mu \mathrm{M}$ ionomycin at $4^{\circ} \mathrm{C}$ for $1 \mathrm{~h}$. After reconstitution and washing, the cells were perfused first with $\mathrm{KRB}$ containing $100 \mu \mathrm{M}$ EGTA and then with $2 \mathrm{mM} \mathrm{Ca}^{2+}$. Until the traces reach steady-state levels. The data are summarized in histograms and expressed as mean \pm SEM of $\mu \mathrm{M}$ steady-state $\left[\mathrm{Ca}^{2+}\right](p=0.0020)$ and areas under the curves (AUC) $(p=0.0011)$ of 16 coverslips from four independent experiments. c WT- and 3Tg-iAstro cells, stably expressing MIT-AEQ Ca ${ }^{2+}$ sensor, were reconstituted with coelenterazine wt (native coelenterazine) in complete culture medium for $1 \mathrm{~h}$. After reconstitution and washing, the cells were perfused first with $\mathrm{KRB}$ containing $2 \mathrm{mM} \mathrm{Ca}^{2+}$. When indicated, perfusion was switched to KRB supplemented with $2 \mathrm{mM} \mathrm{Ca}^{2+}$ and $20 \mu \mathrm{M}$ ATP. The data are expressed as mean \pm SEM $\mu \mathrm{M}$ of peak of $\left[\mathrm{Ca}^{2+}\right](p=$ $0.013)$ and of the $\operatorname{AUC}(p=0.197)$ from 20 coverslips from four independent experiments (biological replicates).

\section{Discussion}

In the present contribution we took advantage of the recently generated $\mathrm{AD}$ astrocytic cellular model, immortalized hippocampal astrocytes from 3xTg-AD mice (3TgiAstro and WT-iAstro cells) to investigate the possible association between astrocytic bioenergetic status, ER and mitochondrial $\mathrm{Ca}^{2+}$ homeostasis and proteostasis, which includes protein synthesis and degradation machinery.
We found that in 3Tg-iAstros (i) glycolysis, OCR and ECAR are significantly lower compared to WT-iAstros, (ii) intracellular and intramitochondrial ROS production is elevated, however (iii) the changes are not due to alterations in expression of mitochondrial proteins, but rather due to extra-mitochondrial protein expression changes, resulting in (iv) ER $\mathrm{Ca}^{2+}$ overload and reduced ATP-evoked mitochondrial $\mathrm{Ca}^{2+}$ transients; (v) increase 


\section{a}
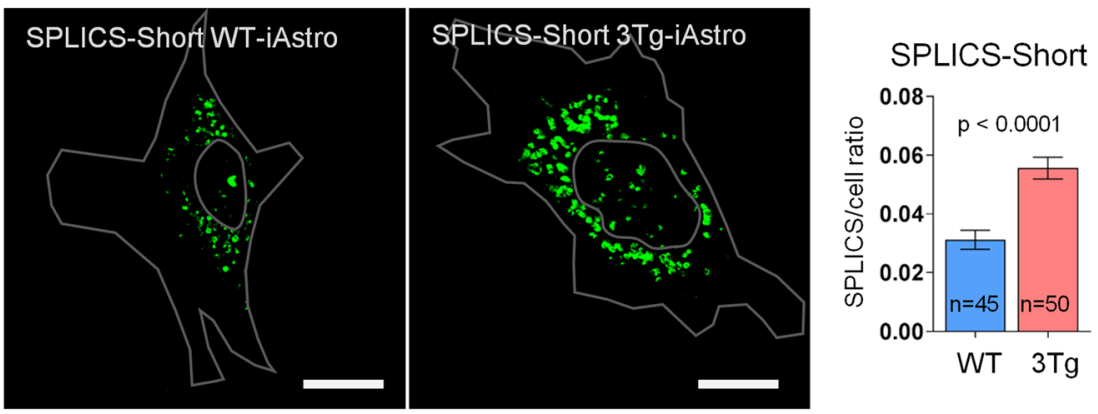

b
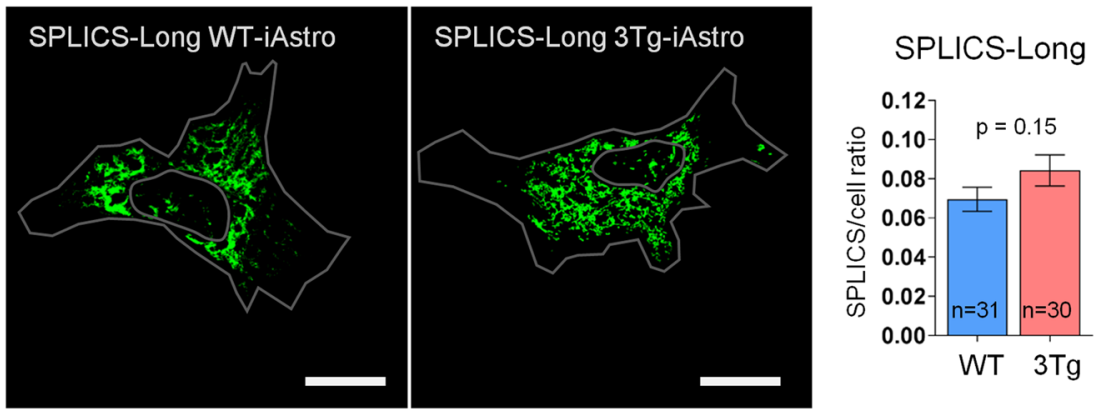

Fig. 7 Increased short-distance mitochondrial-ER tethering in 3Tg-iAstro as compared with WT-iAstro cells. a SPLICS-Short ( $\approx 8-10$ nm) fluorescence in WT-iAstro (left panel) and in 3Tg-iAstro cells (right panel). For each cell fluorescence area was normalized to cell surface area and expressed as mean \pm SEM of $n=45$ (WT-iAstro) and $n=50$ (3Tg-iAstro), $p<0.0001$. b SPLICS-Long ( $\approx 40-50 \mathrm{~nm})$ fluorescence in WT-iAstro (left panel) and in 3Tg-iAstro cells (right panel). Data expressed as mean \pm SEM SPLICS-Long/cell area of $n=31$ (WT-iAstro) and $n=30$ (3Tg-iAstro), $p=0.15$. With gray contour is depicted area of the cell and the nucleus. Bar, $20 \mu \mathrm{m}$.

of area with a shorter $(8-10 \mathrm{~nm})$ mitochondria-ER interaction distance; (vi) increased expression of ER-stress/ UPR-associated genes; and (vii) reduced protein synthesis rate in 3Tg-iAstro compare with WT-iAstro cells.

Two glycolytic proteins were found to be changed in our dataset collected on whole-cell iAstro cells, namely fructose-bisphosphate aldolase A (Aldoa) and isoform M1 of pyruvate kinase PKM $(\mathrm{Pkm})$. Aldoa catalyzes the reversible conversion of fructose-1,6-bisphosphate to glyceraldehyde 3-phosphate and dihydroxyacetone phosphate and is critically involved in glycogen storage: deficiency of this enzyme causes Glycogen Storage Disease XII leading to excessive liver accumulation of glycogen ${ }^{38}$. Therefore, increase of Aldoa expression in 3Tg-iAstro cells may potentially lead to the depletion of glycogen from astrocytes which would reduce bioenergetic reserve of the AD CNS. The second enzyme, Pkm, a member of pyruvate kinases (PK) family, catalyzes the last, and ratelimiting, step of glycolysis by the transfer of a phosphoryl group from phosphoenolpyruvate to ADP generating ATP and pyruvate ${ }^{38}$. Downregulated glycolytic response to mitochondrial stress was also confirmed in human iPSCderived astrocytes with PS1- $\Delta$ E9 mutations ${ }^{39}$. Astrocytes have significantly higher glycolytic metabolism than neurons through which they feed neurons, store glycogen and metabolize and recycle neurotransmitters ${ }^{40,41}$. Slowing down astrocytic glycolysis may have detrimental effects on neuronal activity and survival.

$\mathrm{Ca}^{2+}$ is an important regulator of mitochondrial oxidative metabolism and is required for the activity of enzymes of the tricarboxylic acid cycle, the proteins of the electron transport chain, and the ATP synthase ${ }^{42,43}$. In these terms, the decrease of mitochondrial $\mathrm{Ca}^{2+}$ may explain the reduced OCR and respiratory capacity of 3TgiAstro cells. Moreover, our results are in line with those obtained on APPswe/PS1A246E-expressing astroglioma cells in which reduced mitochondrial OCR was accompanied by insignificant change in mitochondrial $\mathrm{Ca}^{2+}$ in front of strongly potentiated ATP-induced cytosolic $\mathrm{Ca}^{2+}$ increase $^{44}$. However, it has been reported that in iPSCderived astrocytes form patients bearing PS1- $\Delta \mathrm{E} 9$ mutation basal mitochondrial respiration was increased ${ }^{39}$. Such discrepancies may arise from differences in generation of cell models as well as from different AD-related mutations.

A growing body of evidence suggests that the mitochondrial-ER interaction, in terms of both distance and protein composition, may be central for deregulation 

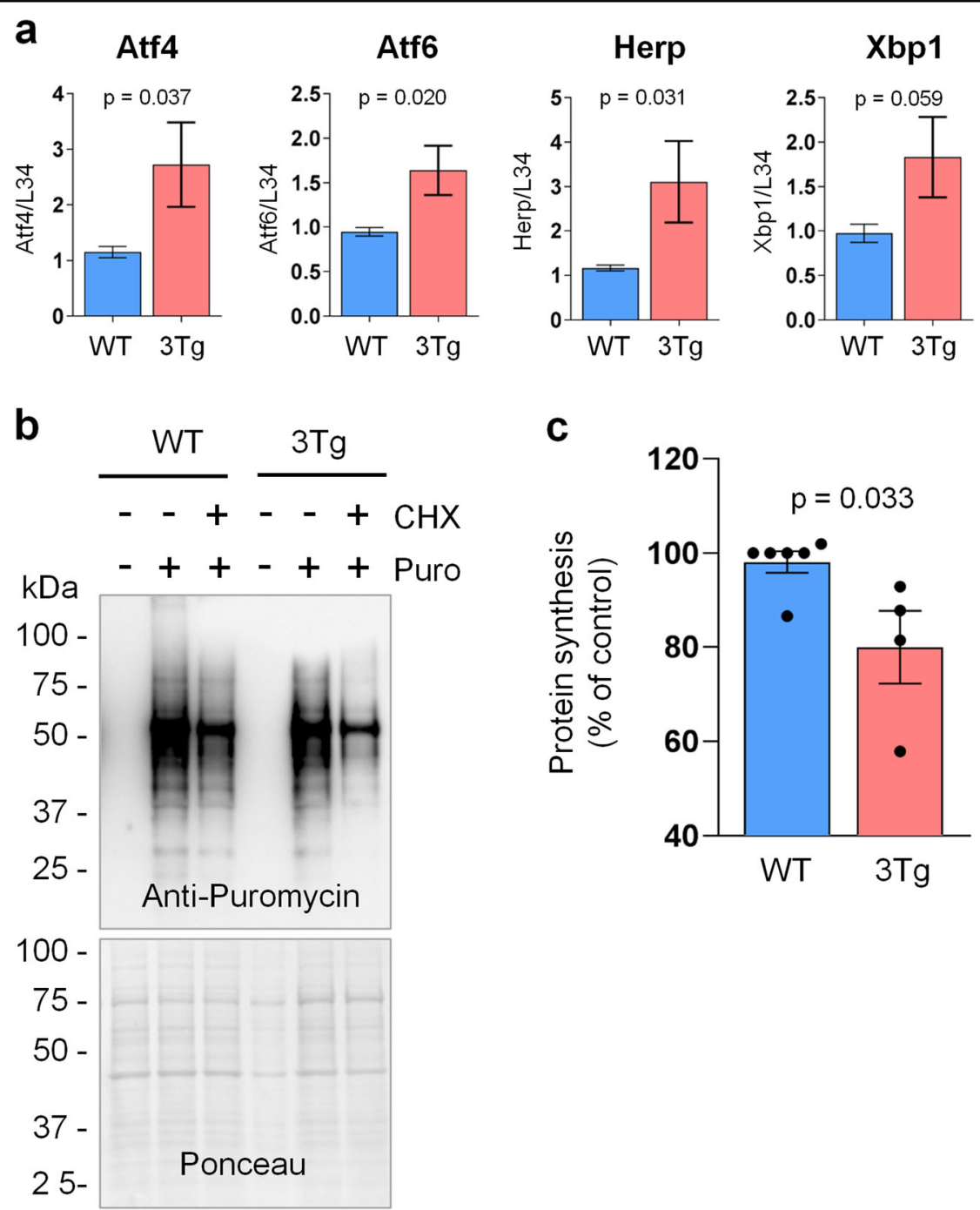

Fig. 8 Activation of ER-stress and reduction of protein synthesis in 3Tg-iAstro vs WT-iAstro cells. a Real-time PCR of ER-stress/UPR responserelated genes Atf4 $(p=0.037)$, Atf6 $(p=0.02)$, Herp $(p=0.031)$ and Xbp1 $(p=0.059)$ of 3Tg-iAstro vs WT-iAstro cells. Data are expressed as mean \pm SEM $\triangle C(t), n=14$ for WT-iAstro, $n=12$ biological replicates for 3Tg-iAstro cells. $\mathbf{b}$ WT and 3 Tg-iAstro were pulsed with puromycin for $3 \mathrm{~h}$. Where indicated, cells were also treated with cycloheximide $(\mathrm{CHX}, 10 \mu \mathrm{M})$ ten minutes before adding puromycin. Anti-puromycin antibody was used to detect neo-synthesized peptides. Ponceau staining was used for band normalization. c Quantification of anti-puromycin detected bands. Data are expressed as mean \pm SEM, six and four independent cultures were used for WT and Tg-iAstro, respectively ( $p=0.033$; two-tailed unpaired Students's $t$ test with Mann-Whitney correction).

of $\mathrm{Ca}^{2+}$ signaling, mitochondrial functions and ERstress $^{14,35,45,46}$. It has been found that the distance between ER and mitochondria is kept in a range of $\approx 10-50 \mathrm{~nm}^{13}$. Increase as well as decrease of this distance affects multiple aspects of mitochondrial functions: $\mathrm{Ca}^{2+}$ signaling, lipid transfer and biogenesis, protein synthesis and transport. We found that in 3Tg-iAstro cells the short distance $(\approx 8-10 \mathrm{~nm})$ sites area is increased by $77 \%$. Although at the moment we cannot draw cause-effect relationships between the mito-ER contact sites shortening and the other findings of this work, we propose a hypothetical mechanistic sequence of events which may occur in astrocytes and has in its center FAD mutationsinduced increase in mito-ER tethering at a short distance. First of all it explains reduced mitochondrial $\mathrm{Ca}^{2+}$ signals in concomitance with ER $\mathrm{Ca}^{2+}$ overload. The mito-ER distance shorter than $10-12 \mathrm{~nm}$ creates steric encumbrance for $\mathrm{IP}_{3} \mathrm{Rs}$ which hampers $\mathrm{Ca}^{2+}$ transfer from $\mathrm{IP}_{3} \mathrm{Rs}$ to mitochondria ${ }^{13}$. Reduced mitochondrial $\mathrm{Ca}^{2+}$ signals may lead to reduced OXPHOS efficiency and ATP production $^{42}$, while cellular $\mathrm{Ca}^{2+}$ overload may be a cause for ER-stress $/ \mathrm{UPR}^{47}$. Both mitochondrial and ER-related abnormalities due to increased agonist-induced $\mathrm{Ca}^{2+}$ release and impaired supply of ATP may lead to global 


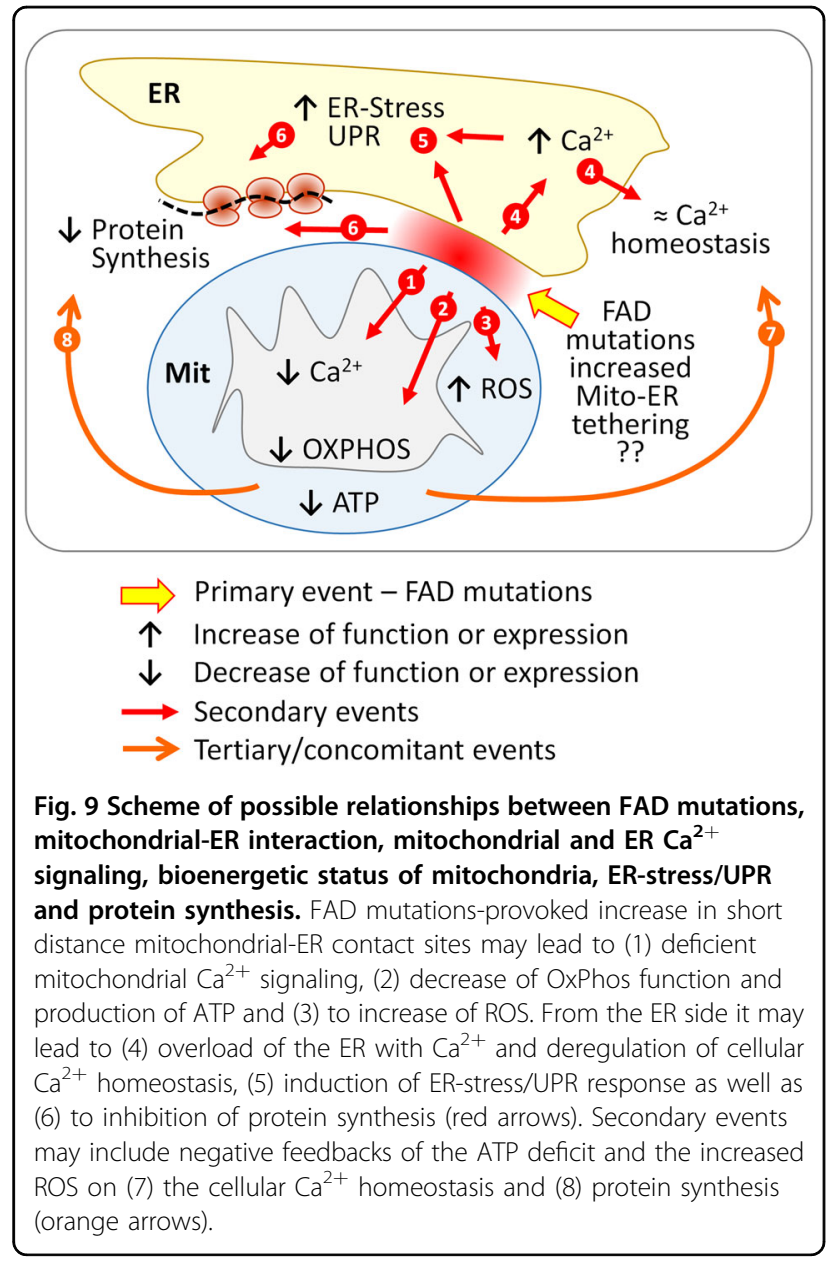

deregulation of cellular $\mathrm{Ca}^{2+}$ homeostasis. At the same time ER-stress and energy deficit may slow down protein synthesis and degradation machinery as these processes are among the most energy consuming in the cell. While details of this hypothetic scenario, illustrated in Fig. 9, are still to be elucidated, recent publication corroborate our results. It has been show that mito-ER tethering in a short mito-ER distance range is augmented in both presenilin 2 N141I mutant human fibroblasts ${ }^{15}$ and upon overexpression of AD-relevant caspase 3-cleaved WT Tau, which is localized specifically at mito-ER contact $\operatorname{sites}^{48}$.

\section{Conclusions}

In the CNS, neuronal performance, survival and resilience in stressful or diseased conditions strongly depends on homeostatic support of astroglial cells ${ }^{19}$. During AD pathogenesis, astrocytes, in their turn, undergo complex, brain region-specific and disease stage-dependent remodeling $^{21,49}$. Our data suggest that these changes concern multiple aspects of astroglial biology, characteristic for $\mathrm{AD}$ as the deregulation of cellular bioenergetics and $\mathrm{Ca}^{2+}$ signaling, mitochondria-ER interaction and proteostasis.
The effects of these changes, in turn, may have multiple consequences, e.g., we recently have shown that conditioned medium from $3 \times \mathrm{Tg}-\mathrm{AD}$ astrocytes reduces synaptic proteins in cultured neurons ${ }^{50}$, while $3 \mathrm{Tg}$ iAstros fail to support the integrity of the blood-brain barrier in vitro ${ }^{51}$. In light of this, our work exemplifies the importance of multidisciplinary approach in studying such complex phenomena as AD and illustrates the use of iAstro cells as versatile and cost-effective astrocytic cellular model of AD. To conclude, our results highlight the important role that astrocytes play in $\mathrm{AD}$ pathogenesis and suggest that the alterations in their homeostatic capabilities contribute to AD progression at early stages of the disease.

\section{Material and methods}

Immortalized hippocampal astrocytes from 3xTg-AD mice

Generation of immortalized astrocytes from hippocampi of WT and 3xTg-AD mice (WT- and 3Tg-iAstro cells) was described elsewhere ${ }^{27}$. iAstro lines were maintained in complete culture media containing Dulbecco's modified Eagle's medium (DMEM; Sigma-Aldrich, Cat. No. D5671) supplemented with $10 \%$ fetal bovine serum (Gibco, Cat. No. 10270), $2 \mathrm{mM}$ L-glutamine (SigmaAldrich), and 1\% penicillin/streptomycin solution (SigmaAldrich). Cells were passaged once a week and used for experiments between passages 12 and 20 from establishment ${ }^{27}$.

\section{Evaluation of iAstro energetic metabolism and ROS production \\ Mitochondrial respiration}

Assessment of mitochondrial and glycolytic functionality was performed by Seahorse XFp Analyser (Agilent Technologies, Santa Clara, CA, USA) using Seahorse XFp Cell Mito Stress Test Kit (Agilent Technologies) and Seahorse XFp Real-Time ATP Rate Assay Kit (Agilent Technologies) according to manufacturer's instructions. Briefly, iAstro cells were seeded into Agilent Seahorse XFp well plates at a density of 100,000 cells $/ \mathrm{cm}^{2}$ and kept in complete culture medium (described in 4.1.) in incubator for 14-16 h. One hour before the measurement, the medium was replaced with Seahorse XF Assay Medium supplemented with $2 \mathrm{mM}$ L-glutamine, $1 \mathrm{mM}$ sodium pyruvate and $10 \mathrm{mM}$ glucose and cells were placed to non- $\mathrm{CO}_{2}$ incubator. Just before the measurement, medium was changed again to the fresh Assay Medium with the same supplements. Final inhibitor concentrations in the wells were $1.5 \mu \mathrm{M}$ oligomycin, $1 \mu \mathrm{M}$ carbonyl cyanide4-phenylhydrazone (FCCP), $0.5 \mu \mathrm{M}$ antimycin A, and $0.5 \mu \mathrm{M}$ rotenone. Oxygen consumption rate (OCR) and extracellular acidification rate (ECAR) were normalized to total cellular protein content determined directly in the plate immediately after each experimental run by 
Bradford assay. Optical density after reaction with the Bradford reagent (Cat. No. B6916, Merck) was assessed by a multifunctional plate reader Infinite 200 Pro M Nano Plex (Tecan, Männedorf, Switzerland). The data were analyzed and single run reports were generated by Wave software (Agilent Technologies), and graphical images of summary data were created by SigmaPlot vs.13 (Systat Software, Slough, UK).

\section{Reactive oxygen species determination}

For intracellular ROS measurement, cells were seeded in black (for plater reader) and transparent (for microscopy) 96 -well plates at a density of $60,000 \mathrm{cells} / \mathrm{cm}^{2}$ and grown for $48 \mathrm{~h}$ in the conditions described in 4.1. After three washes with $37^{\circ} \mathrm{C} 200 \mu$ l Hank's Balanced Salt Solution with $\mathrm{Ca}^{2+}$ and $\mathrm{Mg}^{2+}$ (HBSS/Ca/Mg, Thermo Fisher Scientific), the cells were incubated with $10 \mu \mathrm{M}$ 2',7'-dichlorofluorescin diacetate (DCFDA, Cat. No. D6883, Sigma-Aldrich) in $\mathrm{HBSS} / \mathrm{Ca} / \mathrm{Mg}$ in the dark at $37^{\circ} \mathrm{C}$ for $30 \mathrm{~min}$. After incubation, not incorportated DCFDA is washed away by three times replacing fresh $200 \mu \mathrm{l} 37^{\circ} \mathrm{C}$-warm $\mathrm{HBSS} / \mathrm{Ca} / \mathrm{Mg}$. After last wash, the fluorescence was read in a multifunctional plate reader Infinite 200 Pro M Nano Plex (Tecan, Männedorf, Switzerland) at excitation/emission wavelengths $485 \mathrm{~nm} /$ $535 \mathrm{~nm}$. The images of DCFDA-loaded iAstro cells were also taken under fluorescent microscope Zeiss Axio Observer.Z1 (Carl Zeiss, Oberkochen, Germany) and fluorescence intensity of the cells (excluding cell uncovered areas) was evaluated by ImageJ freeware.

\section{Mitochondrial superoxide determination}

For evaluation of mitochondrial superoxide, the cells were grown in clear 96-well plates the same way as for intracellular ROS assessment. $48 \mathrm{~h}$ after plating, the cells were $3 \mathrm{x}$ washed with HBSS and incubated with $2 \mu \mathrm{M}$ MitoSox $^{\mathrm{TM}}$ Red (Cat. No. M36008, Thermo Fisher Scientific) in HBSS at $37^{\circ} \mathrm{C}$ in the dark for $15 \mathrm{~min}$. The images were taken by fluorescent microscope Zeiss Axio Observer.Z1 and fluorescence intensity in the images was evaluated by ImageJ software. For higher magnification images for monitoring MitoSox staining colocalization with mitochondrial network, the cells were grown on glass-bottom Petri dishes (Catalog No. 0030740009, Eppendorf). For visualization of total mitochondrial network, $500 \mathrm{nM}$ MitoTracker Green FM (Cat. No. M7514, Thermo Fisher Scientific) and for nuclear chromatin staining, $6 \mu \mathrm{g} / \mathrm{ml}$ Hoechst33342 (SigmaAldrich) was added to incubation together with MitoSox prior to microscope imaging.

\section{Mitochondrial membrane potential determination}

Variation in mitochondrial membrane potential $(\Delta \psi \mathrm{m})$ is an important parameter of mitochondrial function and is an indicator of cell health. Mitochondrial membrane potential $(\Delta \psi \mathrm{m})$ was measured by using $5,51,6,61$-tetrachloro-1,11,3,31 tetraethylbenzimidazolyl carbocyanine iodide (JC-1; Cayman, Ann Arbor, Michigan, USA) according to manufacturer's instructions as has been previously describes ${ }^{52,53}$. Briefly, WT- and 3Tg-iAstro cells were plated (10 000 cells/well in 96-well plate) $24 \mathrm{~h}$ before measurement. Then the cells were incubated with JC-1 diluted in Assay Buffer for $15 \mathrm{~min}$ at $37^{\circ} \mathrm{C}$. After washing (twice with Assay Buffer), the mitochondrial membrane potential was determined by measuring the red (excitation $535 \mathrm{~nm} /$ emission $595 \mathrm{~nm}$ ) and green (excitation $485 \mathrm{~nm} /$ emission $535 \mathrm{~nm}$ ) fluorescence using a spectrophotometer $\left(\mathrm{VICTOR}^{\mathrm{TM}} \mathrm{X}\right.$ Multilabel Plate Reader, PerkinElmer). $\Delta \psi \mathrm{m}$ was calculated as ratio of red/green fluorescence intensities and the data are expressed as a fold change of WT-iAstro cells.

\section{Preparation of mitochondria-ER enriched fractions}

WT-iAstro and Tg-iAstro were plated at concentration of $0.5 \times 10^{\wedge} 6$ cells/dish in $10 \mathrm{~cm}$ Petri dishes (50 dishes per line) and grown until 80-90\% confluence. On the day of experiment, the cells were washed twice with PBS, detached with trypsin, counted and collected $20 \times 10^{\wedge} 6$ cells/tube. Fractionation was performed using (Cat. 89874, ThermoFisher, Milan, Italy) according to manufacturer's instructions. Final pellets containing mitochondrial-ER enriched fraction (MERE fraction) were lysed with $50 \mu \mathrm{L}$ of lysis buffer (50 mM TrisHCl (pH 7.4), sodium dodecyl sulphate (SDS) $0.5 \%, 5 \mathrm{mM}$ EDTA), $5 \mu \mathrm{L}$ of protease inhibitors cocktail (PIC, Calbiochem). After quantification (QuantiPro BCA Assay Kit, QPBSA-1kit, ThermoFisher, Milan, Italy), MERE fractions were snap frozen and stored at $-80^{\circ} \mathrm{C}$. Four independently prepared samples of each, WT-iAstro and Tg-iAstro, were subjected to proteomic analysis.

\section{Shotgun mass spectrometry proteomics}

Cell lysates were reduced using $2.5 \mu \mathrm{L}$ of dithiothreitol (200 mM DTT stock solution) (Sigma) at $90^{\circ} \mathrm{C}$ for $20 \mathrm{~min}$, and alkylated with $10 \mu \mathrm{l}$ of Cysteine Blocking Reagent (Iodoacetamide, IAM, $200 \mathrm{mM}$ Sigma) for $1 \mathrm{~h}$ at room temperature in the dark. Trypsin (Promega, Sequence Grade) was added and digestion was performed overnight at $37{ }^{\circ} \mathrm{C}$. Then, peptides digests were desalted on the Discovery ${ }^{\circledR}$ DSC-18 solid-phase extraction (SPE) 96-well Plate $(25 \mathrm{mg} /$ well) (Sigma-Aldrich Inc., St. Louis, MO, USA) and the samples were ready for the analysis ${ }^{27}$.

LC-MS/MS analyses were performed using a micro-LC Eksigent Technologies (Dublin, USA) system with a stationary phase of a Halo Fused C18 column $(0.5 \times 100 \mathrm{~mm}$, $2.7 \mu \mathrm{m}$; Eksigent Technologies, Dublin, USA). The mobile phase was a mixture of $0.1 \%(\mathrm{v} / \mathrm{v})$ formic acid in water $(\mathrm{A})$ and $0.1 \%(\mathrm{v} / \mathrm{v})$ formic acid in acetonitrile (B), eluting at a flow-rate of $15.0 \mu \mathrm{L} \mathrm{min}^{-1}$ at an increasing concentration 
of solvent B from 2 to $40 \%$ in 30 min. Samples used to generate the SWATH-MS (Sequential window acquisition of all theoretical mass spectra) spectral library were subjected to the traditional data-dependent acquisition (DDA): the mass spectrometer analysis was performed using a mass range of 100-1500 Da (TOF scan with an accumulation time of $0.25 \mathrm{~s}$ ), followed by a MS/MS product ion scan from 200 to $1250 \mathrm{Da}$ (accumulation time of $5.0 \mathrm{~ms}$ ) with the abundance threshold set at $30 \mathrm{cps}(35$ candidate ions can be monitored during every cycle). Samples were then subjected to cyclic data independent analysis (DIA) of the mass spectra, using a 25-Da window. A 50-ms survey scan (TOF-MS) was performed, followed by MS/MS experiments on all precursors. These MS/MS experiments were performed in a cyclic manner using an accumulation time of $40 \mathrm{~ms}$ per 25-Da swath (36 swaths in total) for a total cycle time of $1.5408 \mathrm{~s}$. The ions were fragmented for each MS/MS experiment in the collision cell using the rolling collision energy. The MS data were acquired with Analyst TF 1.7 (SCIEX, Concord, Canada). Three instrumental replicates for each sample were subjected to the DIA analysis ${ }^{54,55}$.

The mass spectrometry files were searched using Protein Pilot (AB SCIEX, Concord, Canada) and Mascot (Matrix Science Inc., Boston, USA). Samples were input in the Protein Pilot software v. 4.2 (AB SCIEX, Concord, Canada), with the following parameters: cysteine alkylation, digestion by trypsin, no special factors and False Discovery Rate at $1 \%$. The UniProt Swiss-Prot reviewed database containing mouse proteins (version 12/10/2018, containing 25137 sequence entries). The Mascot search was performed on Mascot v. 2.4, the digestion enzyme selected was trypsin, with two missed cleavages and a search tolerance of $50 \mathrm{ppm}$ was specified for the peptide mass tolerance, and 0.1 Da for the MS/MS tolerance. The charges of the peptides to search for were set to $2+, 3+$ and $4+$, and the search was set on monoisotopic mass. The instrument was set to ESI-QUAD-TOF and the following modifications were specified for the search: carbamidomethyl cysteines as fixed modification and oxidized methionine as variable modification.

The quantification was performed by integrating the extracted ion chromatogram of all the unique ions for a given peptide. The quantification was carried out with PeakView 2.0 and MarkerView 1.2. (Sciex, Concord, ON, Canada). Six peptides per protein and six transitions per peptide were extracted from the SWATH files. Shared peptides were excluded as well as peptides with modifications. Peptides with FDR lower than 1.0\% were exported in MarkerView for the $t$-test.

\section{Fura-2 calcium imaging}

iAstro lines, grown onto $24 \mathrm{~mm}$ round coverslips $\left(3 \times 10^{4}\right.$ cell/coverslip), were loaded with $2.5 \mu \mathrm{M}$ Fura-2/AM (Cat.
No. F1201, Life Technologies, Milan, Italy) in the presence of $0.005 \%$ Pluronic F-127 (Cat. No. P6867, Life Technologies) and $10 \mu \mathrm{M}$ sulfinpyrazone (Cat. S9509, Sigma) in KRB solution ( $125 \mathrm{mM} \mathrm{NaCl}, 5 \mathrm{mM} \mathrm{KCl}, 1 \mathrm{mM}$ $\mathrm{Na}_{3} \mathrm{PO}_{4}, 1 \mathrm{mM} \mathrm{MgSO}$, $5.5 \mathrm{mM}$ glucose, $20 \mathrm{mM}$ HEPES, $\mathrm{pH}$ 7.4) supplemented with $2 \mathrm{mM} \mathrm{CaCl}$. After loading (30 min in a dark place) cells were washed once with KRB solution and allowd to de-esterify for $30 \mathrm{~min}$. After this the coverslips were mounted in an acquisition chamber and placed on the stage of a Leica epifluorescence microscope equipped with a $S$ Fluor $40 \times / 1.3$ objective. Cells were alternatively excited at $340 / 380 \mathrm{~nm}$ by the monochromator Polichrome V (Till Photonics, Munich, Germany) and the fluorescent signal was collected by a CCD camera (Hamamatsu, Japan) through bandpass $510 \mathrm{~nm}$ filter. The fluorescent signals were captured by MetaFluor (Molecular Devices, Sunnyvale, CA, USA) software. The cells were stimulated by $20 \mu \mathrm{M}$ ATP. To quantify the difference in the amplitude of $\mathrm{Ca}^{2+}$ transients, the ratio values were normalized according to the formula $(\Delta \mathrm{F}) / \mathrm{F} 0$ (referred to as norm. Fura ratio).

\section{Generation of stable iAstro lines expressing MIT-AEQ and ER-AEQ}

For measurement of $\mathrm{Ca} 2+$ inside the ER and mitochondria, either mitochondrially or ER-targeted photoprotein aequorin (MIT-AEQ and ER-AEQ probes, respectively) were used. The details of generation of producation of lentiviral vectors expressing aequorin $\mathrm{Ca}^{2}$ ${ }^{+}$probes is described elswhere ${ }^{34} .24 \mathrm{~h}$ after plating (10E4 cell/well in 24-well plate), WT- and Tg-iAstros were infected with lentiviral particles experssing MIT-AEQ and ER-AEQ at MOI from 5 to 20. The dilution of virus that gave more than $50 \%$ of infected cells, as was detected by fluorescence of reporter proteins ${ }^{34}$, were further processed. Upon reaching confluence, the cells were expanded, and MIT-AEQ and ER-AEQ expressing WT- and 3Tg-iAstro cells were inriched using fluorescenceactivated cell sorting (S3e Cell Sorter, Bio-Rad, Segrate, Milano). Sorted cells were expanded and frozen until needed.

\section{Aequorin $\mathrm{Ca}^{2+}$ measurements}

For $\mathrm{Ca}^{2+}$ measurement in the ER lumen, WT- and 3TgiAstro cells expressing ER-AEQ $\mathrm{Ca}^{2+}$ sensor were plated on $13 \mathrm{~mm}$ round coverslips placed in 24-well plates $(5 \times$ $10^{4}$ cell/well). $24 \mathrm{~h}$ after plating che cells were reconstituted in modified Krebs-Ringer buffer (KRB, $135 \mathrm{mM}$ $\mathrm{NaCl}, 5 \mathrm{mM} \mathrm{KCl}, 0.4 \mathrm{mM} \mathrm{KH} 2 \mathrm{PO} 4,1 \mathrm{mM} \mathrm{MgSO} 4$, $5.5 \mathrm{mM}$ glucose, $20 \mathrm{mM}$ HEPES (pH 7.4) supplemented with $600 \mu \mathrm{M}$ EGTA, $5 \mu \mathrm{M}$ coelenterazine-n (low lightemitting coelenterazine variant) and $3 \mu \mathrm{M}$ ionomycin (all reagents from Sigma) for $1 \mathrm{~h}$ at $4{ }^{\circ} \mathrm{C}$. After reconstitution the cells were washed three times with KRB containing 
$600 \mu \mathrm{M}$ EGTA and $2 \%$ BSA, followed by three washes with KRB containing $100 \mu \mathrm{M}$ EGTA, after which the coverslips were trans-ferred into perfusion chamber of a custom-built aequorinometer (CAIRN research, UK). The cells were perfused with KRB supplemented with $100 \mu \mathrm{M}$ EGTA at $37^{\circ} \mathrm{C}$. After $3 \mathrm{~min}$ of baseline recording the perfusion solution was switched to KRB supplemented with $2 \mathrm{mM} \mathrm{Ca}^{2+}$ and recording continued until the $\left[\mathrm{Ca}^{2+}\right]$ in the ER did reach the steady-state level. At the end of each experiment, for quantification of the $\mathrm{ER} \mathrm{Ca}^{2+}$ levels, the cells were perfused with distilled water containing $100 \mathrm{mM} \mathrm{Ca}^{2+}$ and $0.1 \%$ Triton X-100 to disrupt cells and discharge the remaining AEQ pool.

For assessment of ATP-induced $\mathrm{Ca}^{2+}$ transients in the mitochondrial matrix, MIT-AEQ-expressing WT- and 3Tg-iAstro cells were reconstituted with coelenterazine wt $(5 \mu \mathrm{M})$ in complete culture medium in $37^{\circ} \mathrm{C}$ and $5 \%$ $\mathrm{CO} 2$ for $1 \mathrm{~h}$. After washing (3 times) with KRB supplemented with $2 \mathrm{mM} \mathrm{Ca}^{2+}$, cells were transferred into perfusion chamber of a custom-built aequorinometer and acquisition started in perfutio with KRB supplemented with $2 \mathrm{mM} \mathrm{Ca}^{2+}$. After baseline recording, perfusion was switched to KRB supplemented with $2 \mathrm{mM} \mathrm{Ca}^{2+}$ and $20 \mu \mathrm{M}$ ATP. At the end of each experiment, for quantification of the ER $\mathrm{Ca}^{2+}$ levels, the cells were perfused with distilled water containing $100 \mathrm{mM} \mathrm{Ca}^{2+}$ and $0.1 \%$ Triton X-100 to disrupt cells and discharge the remaining AEQ pool. The light signals were calibrated off-line into $\left[\mathrm{Ca}^{2+}\right]$ values using an algorithm described in $^{34}$.

\section{Visualization of mitochondria-ER contact sites}

Mitochondria-ER contact sites were visualized using SPLICS probe as described in ${ }^{15}$. Briefly, WT-iAstro and 3Tg-iAstro were plated onto $13 \mathrm{~mm}$ glass coverslips in 24well plates (3x10E4 cells/well). $24 \mathrm{~h}$ after plating cells were transfected with pairs of plasmids SPLICS-Mit-Short + SPLICS-ER (hereafter referred as SPLICS-Short) and SPLICS-Mit-Long + SPLICS-ER (hereafter referred as SPLICS-Long) in 1:1 ratio using Lipofectamine 2000 (Thermofisher, Milan, Italy). $24 \mathrm{~h}$ after transfection cells were washed with PBS and fixed in $4 \%$ formaldehyde (Sigma, Milan, Italy). Fluorescent image stacks were taken using Zeiss 710 confocal laser scanning microscope equipped with Plan-Apochromat 63x/1.40 Oil M27 objective and Zen software. Image stacks were processed offline to major intensity projections. Thresholding and area calculations were done using Fiji package of ImageJ software v.1.52p. Data are expressed as SPLICS/cell area ratio.

\section{RNA extractiona and real-time PCR}

Total RNA was extract by using the TripleXtractor reagent (Bio-Cell) as indicated by the supplier, and the
AMV Reverse Transcriptase kit (Promega) was used to produce cDNA following the manufacturer's recommendations, by using $2 \mu \mathrm{g}$ of total RNA. Quantitative PCR reactions were performed by using the Bio-Rad CFX96 qPCR thermocycler. The primer pair sequences for selected amplicons were designed using the online IDT PrimerQuest Tool software (IDT; https://eu.idtdna.com/ Primerquest/Home/Index). Primer sequences are available in Supplementary Table 1. Gapdh mRNA level was used as an internal control and results were expressed as previously described ${ }^{56}$.

\section{Puromycin incorporation method (Surface sensing of translation, SUnSET)}

iAstro-WT and TG were incubated with $4 \mu \mathrm{M}$ puromycin dihydrochloride (Sigma Cat. P8833) supplemented in normal medium at $37^{\circ} \mathrm{C}$ with $5 \% \mathrm{CO} 2$ for $3 \mathrm{~h}^{36}$. Subsequently, cells lysates were subjected to Western blot assay.

\section{Cell lysis and western blot}

iAstro-cultures were lysed with $50 \mu \mathrm{L}$ of lysis buffer (50 mM TrisHCl (pH 67.4), sodium dodecyl sulphate (SDS) $0.5 \%, 5 \mathrm{mM}$ EDTA), $5 \mu \mathrm{L}$ of protease inhibitors cocktail (PIC, Calbiochem) and $1 \mu \mathrm{L}$ of phosphatase inhibitor $\mathrm{Na}_{3} \mathrm{VO}_{4} 1 \mathrm{M}$; removed with a scraper, and collected in a 1,5 $\mathrm{ml}$ tube. Lysates were then boiled for 5', and quantified with QuantiPro BCA Assay Kit (Sigma, cat. SLBF3463).

$30 \mu \mathrm{g}$ of proteins were mixed with the right amount of Laemmli Sample Buffer 4X (Bio-Rad), and boiled for 7'. Then samples were loaded on a $12 \%$ polyacrylamidesodium dodecyl sulphate gel for electrophoresis. Proteins were transferred onto nitrocellulose membrane, using Mini Transfer Packs or Midi Transfer Packs, with TransBlot ${ }^{\circledR}$ Turbo TM (Bio-Rad) according to manufacturer's instructions (Bio-Rad).

The membrane was blocked in 5\% skim milk (Sigma, Cat. 70166) for $45^{\prime}$ at room temperature. Subsequently, membrane was incubated with indicated primary antibody, overnight at $4{ }^{\circ} \mathrm{C}$ in agitation. Primary antibodies used were: anti-puromycin (1:1000, Millipore, Cat. MABE343), and ponceau staining was used to normalize protein load. Goat anti-mouse IgG $(\mathrm{H}+\mathrm{L})$ horseradisch peroxidase-conjugated secondary antibody (1:5000; Cat. 170-6516, Bio-Rad) and Goat anti-mouse Igg $(\mathrm{H}+\mathrm{L})$ horseradisch peroxidase-conjugated secondary antibody (1:5000; Cat. 170-6515, Bio-Rad). Detection was carried out with SuperSignalTM West Pico PLUS Chemiluminescent Substrate (Thermo Scientific), based on the chemiluminescence of luminol and developed using ChemiDocTM Imaging System (Bio-Rad). Quantitative densitometry of protein bands analysis was performed with ImageLab software. 


\section{Statistical analysis}

The data for mitochondrial and glycolytic efficiency and ROS production (Figs. 1 and 2 except Fig. 2e) are presented as mean $\pm \mathrm{SD}$; statistical analysis was performed by SigmaPlot vs.13 (Systat Software, Slough, UK). Other data (Fig. 2e; Figs. 6-8) are presented as mean \pm SEM and were analyzed using GraphPad Prism v.7. No samples/results were excluded from the analysis. Unpaired two-tailed Student's $t$ test was used in all experiments and differences were considered significant at $p<0.05$.

\section{Acknowledgements}

This work had the following financial supports: grants 2013-0795 to A.A.G., 2014-1094 to D.L. from the Fondazione Cariplo; grants DSF-FAR-2016 and DSFFAR-2019 to D.L. from The Università del Piemonte Orientale; L.T. was supported by fellowship from the CRT Foundation (1393-2017).

\section{Author details}

${ }^{1}$ Department of Pharmaceutical Sciences, Università degli Studi del Piemonte Orientale, Novara, Italy. ${ }^{2}$ Institute of Pharmaceutical Technologies, Faculty of Pharmacy, Medical Academy, Lithuanian University of Health Sciences, Kaunas, Lithuania. ${ }^{3}$ Department of Translational Medicine, University of Piemonte Orientale, Novara, Italy. ${ }^{4}$ Center for Translational Research on Autoimmune and Allergic Diseases (CAAD), University of Piemonte Orientale, Novara, Italy. ${ }^{5}$ Department of Health Science, University of Piemonte Orientale, Novara, Italy. ${ }^{6}$ DiSIT, University of Piemonte Orientale, Alessandria, Italy. ${ }^{7}$ Department of Drug Chemistry, Faculty of Pharmacy, Medical Academy, Lithuanian University of Health Sciences, Kaunas, Lithuania. ${ }^{8}$ Interdisciplinary Research Center of Autoimmune Diseases (IRCAD), University of Piemonte Orientale, Novara, Italy

\section{Conflict of interest}

The authors declare that they have no conflict of interest.

\section{Publisher's note}

Springer Nature remains neutral with regard to jurisdictional claims in published maps and institutional affiliations.

Supplementary Information accompanies this paper at (https://doi.org/ 10.1038/s41419-020-02911-1).

Received: 12 July 2020 Revised: 1 August 2020 Accepted: 6 August 2020 Published online: 18 August 2020

\section{References}

1. Tse, K.-H. \& Herrup, K. Re-imagining Alzheimer's disease - the diminishing importance of amyloid and a glimpse of what lies ahead. J. Neurochem. 143, 432-444 (2017).

2. Alzheimer's Association Calcium Hypothesis Workgroup. Calcium Hypothesis of Alzheimer's disease and brain aging: a framework for integrating new evidence into a comprehensive theory of pathogenesis. Alzheimers Dement 13, 178-182.e17 (2017).

3. Swerdlow, R. H. Mitochondria and mitochondrial cascades in Alzheimer's disease. J. Alzheimers Dis. 62, 1403-1416 (2018).

4. Area-Gomez, E., Guardia-Laguarta, C., Schon, E. A. \& Przedborski, S. Mitochondria, OxPhos, and neurodegeneration: cells are not just running out of gas. J. Clin. Invest. 129, 34-45 (2019).

5. Camandola, S. \& Mattson, M. P. Aberrant subcellular neuronal calcium regulation in aging and Alzheimer's disease. Biochim. Biophys. Acta 1813, 965-973 (2011).

6. Reese, L. C. \& Taglialatela, G. A role for calcineurin in Alzheimer's disease. Curr. Neuropharmacol. 9, 685-692 (2011)

7. Terada, T. et al. In vivo mitochondrial and glycolytic impairments in patients with Alzheimer disease. Neurology 94, e1592-1604 (2020).
8. Yin, F., Sancheti, H., Patil, I. \& Cadenas, E. Energy metabolism and inflammation in brain aging and Alzheimer's disease. Free Radic. Biol. Med. 100, 108-122 (2016).

9. Cenini, G. \& Voos, W. Mitochondria as potential targets in Alzheimer disease therapy: an update. Front Pharm. 10, 902 (2019).

10. Kowalska, M. et al. Genetic Variants and Oxidative Stress in Alzheimer's Disease Curr. Alzheimer Res 17, 208-223 (2020).

11. Tang, J., Oliveros, A. \& Jang, M.-H. Dysfunctional mitochondrial bioenergetics and synaptic degeneration in Alzheimer disease. Int Neurourol. J. 23, S5-S10 (2019).

12. Jadiya, P. et al. Impaired mitochondrial calcium efflux contributes to disease progression in models of Alzheimer's disease. Nat Commun 10, 3885 (2019).

13. Giacomello, M. \& Pellegrini, L. The coming of age of the mitochondria-ER contact: a matter of thickness. Cell Death Differ. 23, 1417-1427 (2016).

14. Naon, D. \& Scorrano, L. At the right distance: ER-mitochondria juxtaposition in cell life and death. Biochim. Biophys. Acta 1843, 2184-2194 (2014).

15. Cieri, D. et al. SPLICS: a split green fluorescent protein-based contact site sensor for narrow and wide heterotypic organelle juxtaposition. Cell Death Differ. 25, 1131-1145 (2018).

16. Korkotian, E., Meshcheriakova, A. \& Segal, M. Presenilin 1 Regulates [Ca2+]i and Mitochondria/ER Interaction in Cultured Rat Hippocampal Neurons. Oxid. Med Cell Longev. 2019, 7284967 (2019).

17. Garrido-Maraver, J., Loh, S. H. Y. \& Martins, L. M. Forcing contacts between mitochondria and the endoplasmic reticulum extends lifespan in a Drosophila model of Alzheimer's disease. Biol Open 9, bio047530 (2020).

18. Verkhratsky, A., Olabarria, M., Noristani, H. N., Yeh, C.-Y. \& Rodriquez, J. J. Astrocytes in Alzheimer's disease. Neurotherapeutics 7, 399-412 (2010).

19. Verkhratsky, A. \& Nedergaard, M. The homeostatic astroglia emerges from evolutionary specialization of neural cells. Philos. Trans. R. Soc. Lond., B, Biol. Sci. 371, 20150428 (2016).

20. Lim, D., Ronco, V., Grolla, A. A., Verkhratsky, A. \& Genazzani, A. A. Glial calcium signalling in Alzheimer's disease. Rev. Physiol. Biochem. Pharm. 167, 45-65 (2014).

21. Lim, D. et al. Calcium signalling toolkits in astrocytes and spatio-tempora progression of Alzheimer's disease. Curr. Alzheimer Res 13, 359-369 (2016).

22. Verkhratsky, A., Zorec, R., Rodríguez, J. J. \& Parpura, V. Astroglia dynamics in ageing and Alzheimer's disease. Curr. Opin. Pharm. 26, 74-79 (2016).

23. Jackson, J. G. \& Robinson, M. B. Regulation of mitochondrial dynamics in astrocytes: Mechanisms, consequences, and unknowns. Glia 66, 1213-1234 (2018).

24. Kim, D. K. \& Mook-Jung, I. The role of cell type-specific mitochondrial dysfunction in the pathogenesis of Alzheimer's disease. BMB Rep. 52, 679-688 (2019).

25. McAvoy, K. \& Kawamata, H. Glial mitochondrial function and dysfunction in health and neurodegeneration. Mol. Cell. Neurosci. 101, 103417 (2019).

26. Lampinen, R., Belaya, I., Boccuni, I. \& Kanninen, T. M. and K. M. Mitochondrial function in Alzheimer's disease: focus on astrocytes. Astrocyte Physiol. Pathol. (2017) https://doi.org/10.5772/intechopen.71825.

27. Rocchio, F. et al. Gene expression, proteome and calcium signaling alterations in immortalized hippocampal astrocytes from an Alzheimer's disease mouse model. Cell Death Dis. 10, 24 (2019).

28. Ruffinatti, F. et al. Transcriptional remodeling in primary hippocampal astrocytes from an Alzheimer's disease mouse model. Curr. Alzheimer Res. (2018) https://doi.org/10.2174/1567205015666180613113924.

29. Brieger, K., Schiavone, S., Miller, F. J. \& Krause, K.-H. Reactive oxygen species: from health to disease. Swiss Med. Wkly 142, w13659 (2012).

30. Fu, Y.-J., Xiong, S., Lovell, M. A. \& Lynn, B. C. Quantitative proteomic analysis of mitochondria in aging PS-1 transgenic mice. Cell. Mol. Neurobiol. 29, 649-664 (2009).

31. $\mathrm{Yu}, \mathrm{H}$. et al. Mitochondrial molecular abnormalities revealed by proteomic analysis of hippocampal organelles of mice triple transgenic for Alzheimer Disease. Front Mol. Neurosci. 11, 74 (2018).

32. Adav, S. S., Park, J. E. \& Sze, S. K. Quantitative profiling brain proteomes revealed mitochondrial dysfunction in Alzheimer's disease. Mol. Brain 12, 8 (2019).

33. Völgyi, $K$. et al. Early presymptomatic changes in the proteome of mitochondria-associated membrane in the APP/PS1 mouse model of Alzheimer's disease. Mol. Neurobiol. 55, 7839-7857 (2018).

34. Lim, D., Bertoli, A., Sorgato, M. C. \& Moccia, F. Generation and usage of aequorin lentiviral vectors for $\mathrm{Ca}(2+)$ measurement in sub-cellular compartments of hard-to-transfect cells. Cell Calcium 59, 228-239 (2016). 
35. Marchi, S. et al. Endoplasmic reticulum-mitochondria communication through Ca2+ signaling: the importance of mitochondria-associated membranes (MAMs). Adv. Exp. Med. Biol. 997, 49-67 (2017)

36. Schmidt, E. K., Clavarino, G., Ceppi, M. \& Pierre, P. SUnSET, a nonradioactive method to monitor protein synthesis. Nat. Methods 6, 275-277 (2009).

37. Dematteis, G. et al. Calcineurin controls expression of EAAT1/GLAST in mouse and human cultured astrocytes through dynamic regulation of protein synthesis and degradation. Int. J. Mol. Sci. (2020) https://doi.org/10.20944/ preprints202003.0234.v1.

38. Tarnopolsky, M. A. Myopathies related to glycogen metabolism disorders. Neurotherapeutics 15, 915-927 (2018).

39. Oksanen, M. et al. PSEN1 mutant iPSC-derived model reveals severe astrocyte pathology in Alzheimer's disease. Stem Cell Rep. 9, 1885-1897 (2017).

40. Bolaños, J. P. Bioenergetics and redox adaptations of astrocytes to neuronal activity. J. Neurochem. 139(Suppl 2), 115-125 (2016).

41. Juaristi, I. et al. The response to stimulation in neurons and astrocytes. Neurochem. Res. 44, 2385-2391 (2019).

42. Tarasov, A. I., Griffiths, E. J. \& Rutter, G. A. Regulation of ATP production by mitochondrial $\mathrm{Ca}(2+)$. Cell Calcium 52, 28-35 (2012).

43. Williams, G. S. B., Boyman, L. \& Lederer, W. J. Mitochondrial calcium and the regulation of metabolism in the heart. J. Mol. Cell. Cardiol. 78, 35-45 (2015).

44. Martin-de-Saavedra, M. D. et al. The APPswe/PS1A246E mutations in an astrocytic cell line leads to increased vulnerability to oxygen and glucose deprivation, $\mathrm{Ca} 2+$ dysregulation, and mitochondrial abnormalities. J. Neurochem. 145, 170-182 (2018).

45. Qi, H. \& Shuai, J. Alzheimer's disease via enhanced calcium signaling caused by the decrease of endoplasmic reticulum-mitochondrial distance. Med. Hypotheses 89, 28-31 (2016).

46. Tagaya, M. \& Arasaki, K. Regulation of mitochondrial dynamics and autophagy by the mitochondria-associated membrane. Adv. Exp. Med. Biol. 997, 33-47 (2017).
47. Tabas, I. \& Ron, D. Integrating the mechanisms of apoptosis induced by endoplasmic reticulum stress. Nat. Cell Biol. 13, 184-190 (2011).

48. Cieri, D. et al. Tau localises within mitochondrial sub-compartments and its caspase cleavage affects ER-mitochondria interactions and cellular Ca2+ handling. Biochim Biophys. Acta Mol. Basis Dis. 1864, 3247-3256 (2018).

49. Verkhratsky, A., Rodrigues, J. J., Pivoriunas, A., Zorec, R. \& Semyanov, A Astroglial atrophy in Alzheimer's disease. Pflug. Arch. https://doi.org/10.1007/ s00424-019-02310-2 (2019).

50. Tapella, L. et al. TGF- $\beta 2$ and TGF- $\beta 3$ from cultured $\beta$-amyloid-treated or 3xTgAD-derived astrocytes may mediate astrocyte-neuron communication. Eur. J. Neurosci. 47, 211-221 (2018).

51. Kriaučiūnaitè, K. et al. Immortalised hippocampal astrocytes from 3xTG-AD mice fail to support BBB integrity in vitro: role of extracellular vesicles in glialendothelial communication. Cell. Mol. Neurobiol. (2020) https://doi.org/ 10.1007/s10571-020-00871-w.

52. De Cillà, S. et al. Aflibercept and ranibizumab modulate retinal pigment epithelial cells function by acting on their cross talk with vascular endothelial cells. Cell. Physiol. Biochem. 54, 161-179 (2020).

53. Farruggio, S. et al. Genistein and $17 \beta$-estradiol protect hepatocytes from fatty degeneration by mechanisms involving mitochondria, inflammasome and kinases activation. Cell. Physiol. Biochem. 54, 401-416 (2020).

54. Martinotti, S. et al. HMGB1 osteo-modulatory action on osteosarcoma SaOS-2 cell line: an integrated study from biochemical and -omics approaches. J. Cell. Biochem. 117, 2559-2569 (2016).

55. Dalla Pozza, E. et al. Trichostatin A alters cytoskeleton and energy metabolism of pancreatic adenocarcinoma cells: an in depth proteomic study. J. Cell. Biochem. 119, 2696-2707 (2018).

56. Gagliardi, M. et al. Aldo-keto reductases protect metastatic melanoma from ER stress-independent ferroptosis. Cell Death Dis. 10, 902 (2019). 